\title{
On the Minimum-Area Rectangular and Square Annulus Problem*
}

\author{
Sang Won Bae
}

$16^{\text {th }}$ April, $2019 \quad 01: 21$

\begin{abstract}
In this paper, we address the minimum-area rectangular and square annulus problem, which asks a rectangular or square annulus of minimum area, either in a fixed orientation or over all orientations, that encloses a set $P$ of $n$ input points in the plane. To our best knowledge, no nontrivial results on the problem have been discussed in the literature, while its minimum-width variants have been intensively studied. For a fixed orientation, we show reductions to well-studied problems: the minimum-width square annulus problem and the largest empty rectangle problem, yielding algorithms of time complexity $O\left(n \log ^{2} n\right)$ and $O(n \log n)$ for the rectangular and square cases, respectively. In arbitrary orientation, we present $O\left(n^{3}\right)$-time algorithms for the rectangular and square annulus problem by enumerating all maximal empty rectangles over all orientations. The same approach is shown to apply also to the minimum-width square annulus problem and the largest empty square problem over all orientations, resulting in $O\left(n^{3}\right)$-time algorithms for both problems. Consequently, we improve the previously best algorithm for the minimum-width square annulus problem by a factor of logarithm, and present the first algorithm for the largest empty square problem in arbitrary orientation. We also consider bicriteria optimization variants, computing a minimum-width minimum-area or minimum-area minimum-width annulus.
\end{abstract}

Keywords: rectangular annulus, square annulus, minimum area, minimum width, arbitrary orientation

\section{Introduction}

An annulus informally depicts a ring-shaped region in the plane, often described by two concentric circles. One can consider a generalization to any convex shape, such as squares and rectangles. Recently, the minimum-width annulus problem has been studied intensively by researchers, in which a set $P$ of $n$ points in the plane is given and one wants to find an annulus of a certain shape with minimum width that encloses the set $P$ of points. This problem can be seen as a typical geometric covering problem that seeks a minimum-size geometric shape that covers a given set $P$ of points. The annulus problem has applications in shape recognition, facility location, and curve fitting.

Among other shapes, the circular annulus problem has been most intensively studied with an application to the roundness problem 14, 21,24. The first sub-quadratic $O\left(n^{\frac{8}{5}+\epsilon}\right)$-time algorithm was presented by Agarwal et al. [3] The currently best exact algorithm takes $O\left(n^{\frac{3}{2}+\epsilon}\right)$ time by Agarwal and Sharir [2]. Linear-time approximation schemes are also known by Agarwal et al. [4] and by Chan [10]. Abellanas et al. [1] considered minimum-width rectangular annuli that are axis-parallel, and presented two algorithms taking $O(n)$ or $O(n \log n)$ time: one minimizes the width over rectangular annuli with arbitrary aspect ratio and the other does

\footnotetext{
*This work was supported by Kyonggi University Research Grant 2018.

${ }^{\dagger}$ Division of Computer Science and Engineering, Kyonggi University, Suwon, Korea. Email: swbae@kgu.ac.kr
} 


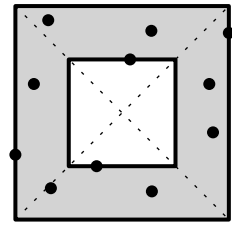

(a)

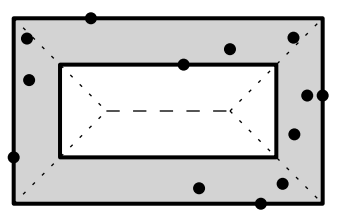

(b)

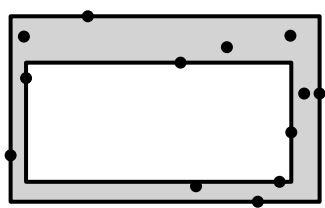

(c)

Figure 1: (a) A square annulus, (b) a uniform rectangular annulus, and (c) a rectangular annulus that is not uniform, enclosing a set of points, all of which are axis-parallel. Each of the three here is of minimum possible width among all square, uniform rectangular, and rectangular annuli, respectively, enclosing the points.

over rectangular annuli with a prescribed aspect ratio, respectively. Gluchshenko et al. 15 presented an $O(n \log n)$-time algorithm that computes a minimum-width axis-parallel square annulus, and proved a matching lower bound, while the second algorithm by Abellanas et al. can do the same in the same time bound. If one considers rectangular or square annuli in arbitrary orientation, the problem becomes more difficult. Mukherjee et al. [18 presented an $O\left(n^{2} \log n\right)$-time algorithm that computes a minimum-width rectangular annulus in arbitrary orientation and arbitrary aspect ratio. The author 7 recently showed that a minimum-width square annulus in arbitrary orientation can be computed in $O\left(n^{3} \log n\right)$ time. Other variants such as the minimum-width annulus problem with outliers 6,8 and the maximum-width empty annulus problem $[9,13$ have also been studied.

Like other geometric covering problems, one may consider several different objective functions to optimize for annuli. Among those objectives, in this paper, we address the problem of finding an annulus of minimum area enclosing the input points $P$, namely, the minimum-area annulus problem. In the literature, it is surprisingly hard to find results on annuli of minimum area, but a few remarks about the minimum-area circular annulus problem. As earlier work pointed out and Chan 10 discussed, the minimum-area circular annulus problem can be formulated into a linear programming, so can be solved in linear $O(n)$ time. It should be mentioned that the currently best known algorithm for the minimum-width circular annulus problem takes $O\left(n^{\frac{3}{2}+\epsilon}\right)$ time [2]. Little is known, however, about rectangular and square annuli of minimum area. The purpose of this paper is thus to gain the understanding of the minimum-area rectangular and square annulus problem and their relations with other well-known geometric problems, and finally to achieve efficient algorithms.

A circular or square annulus is defined by two concentric circles or squares, respectively. However, a rectangular annulus can be defined in several ways, and, in the literature, two different definitions are often introduced. In either case, a rectangular annulus is the closed region between two side-parallel rectangles $R$ and $R^{\prime}$ such that $R^{\prime} \subseteq R$. One may restrict the inner rectangle $R^{\prime}$ to be an offset of the outer rectangle $R$ so that the distance from any point in the boundary of $R^{\prime}$ to the boundary of $R$ is all uniform [1,8, while he/she may allow any rectangle contained in $R$ to be the inner rectangle $R^{\prime}$ [18]. In this paper, we call the latter a rectangular annulus in general and the former a uniform rectangular annulus. See Figure 1 for an illustration. It is not difficult to see that these two definitions of rectangular annuli are equivalent for the minimum-width problem and other variants, while this is not the case for the minimum-area problem.

In this paper, we present first efficient algorithms for the problem of computing a minimumarea square annulus or rectangular annulus, either in a fixed or over all orientations. For the 
Table 1: Summary on the currently best algorithms for the square or rectangular annulus problem. Each column of the table marked by 'W', 'A', 'AW', and 'WA' stands for 'minimum-width', 'minimum-area', 'minimum-area minimum-width', and 'minimum-width minimum-area', respectively. In each row, 'f', 'a', 'S', 'uR', and 'R' are abbreviations for 'square annulus', 'uniform rectangular annulus', 'rectangular annulus', 'in a fixed orientation', and 'in arbitrary orientation', respectively. For each case, the table indicates the wort-case time complexity of the currently best algorithm, omitting the big-Oh $O(\cdot)$ symbol, and its reference. Details on the parameters $r=O\left(n^{2}\right)$ and $t=O\left(n^{2}\right)$ can be found in Theorems 5 and 8 , respectively.

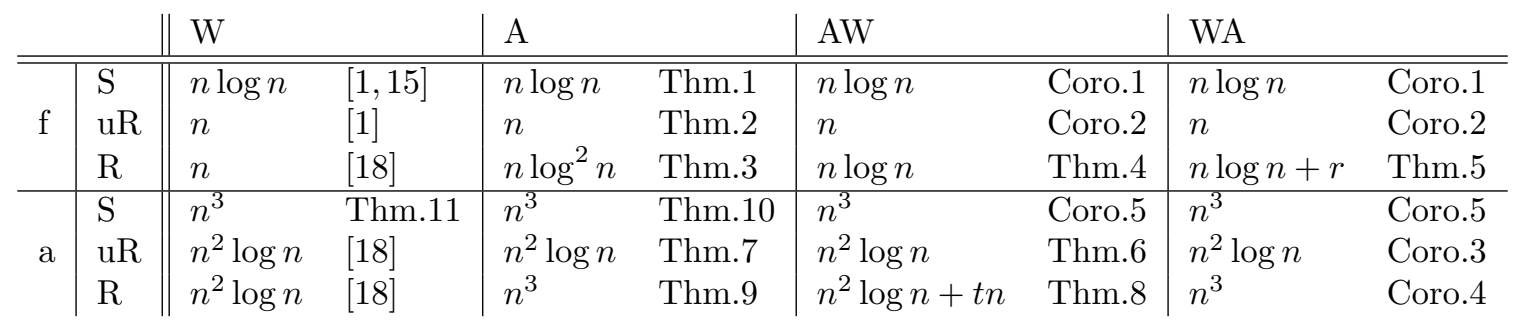

purpose, we show some relations between the minimum-area problem and other well-known geometric optimization problems, such as the minimum-width annulus problem and the largest empty rectangle problem. As a result, the minimum-area problem for square and rectangular annuli is as hard as the minimum-width variant. It is interesting to note that it is not the case for circular annuli. We also consider the bicriteria optimization problems: computing a minimum-area minimum-width annulus or a minimum-width minimum-area annulus.

Our results are summarized as follows.

- In a fixed orientation, a minimum-area square or uniform rectangular annulus enclosing a given set $P$ of $n$ points is shown to be one of also minimum width. On the other hand, we show that existing algorithms [1, 15] for the minimum-width variant indeed compute an annulus of minimum area.

- We show that, in a fixed orientation, the minimum-area rectangular annulus problem and the largest empty rectangle problem are reduced to each other in linear time. The largest empty rectangle problem is known to have a lower bound of $\Omega(n \log n)$ [17. Hence, any algorithm computing a largest empty rectangle can be used to compute a minimum-area rectangular annulus in the same time bound.

- A minimum-area uniform rectangular annulus enclosing $P$ in arbitrary orientation can be computed in $O\left(n^{2} \log n\right)$ time by modifying the algorithm of Mukherjee et al. 18 .

- A minimum-area rectangular annulus enclosing $P$ in arbitrary can be computed in $O\left(n^{3}\right)$ time by enumerating all maximal empty rectangles among $P$ in all orientations using the algorithm by Chaudhuri et al. [11].

- The above approach for the minimum-area rectangular annulus problem is shown to apply to other variants, resulting in the same time bound $O\left(n^{3}\right)$ : computing a minimum-area square annulus, computing a minimum-width square annulus, and computing a largest empty square among $P$ in arbitrary orientation. Remarkably, for the minimum-width square annulus problem, we improve the previously best algorithm [7] of running time $O\left(n^{3} \log n\right)$ by a factor of logarithm; for the largest empty square problem, no discussion can be found in the literature and therefore we present the first efficient algorithm.

- We present first algorithms for some bicriteria variants of the problem: computing a minimum-area minimum-width annulus or a minimum-width minimum-area annulus. 
Table 1 summarizes the running times of the currently best algorithms for the \{minimum-width, minimum-area, minimum-area minimum-width, minimum-width minimum-area\} \{square, uniform rectangular, rectangular\} annulus problem in \{fixed, arbitrary\} orientation.

The rest of the paper is organized as follows: after introducing necessary preliminaries in Section 2, we consider the axis-parallel case of the problem in Section 3 . We then address the problem of rectangular annuli in arbitrary orientation in Section 4, and square annuli in Section 5. We finally conclude the paper by Section 6 with some remarks and open questions.

\section{Preliminaries}

In this paper, we consider the plane $\mathbb{R}^{2}$ with a standard coordinate system, having the horizontal $x$-axis and the vertical $y$-axis. For any subset $A \subseteq \mathbb{R}^{2}$, its boundary and interior, denoted by $\partial A$ and $\operatorname{int} A$, are defined from the standard topology on $\mathbb{R}^{2}$. For any two points $p, q \in \mathbb{R}^{2}$, let $\overline{p q}$ denote the line segment joining $p$ and $q$, and $|\overline{p q}|$ denote the Euclidean length of $\overline{p q}$.

Orientations. The orientation of a line or line segment $\ell$ in the plane is a real number $\theta$ in range $[0, \pi)$ such that the rotated copy of the $x$-axis by $\theta$ counter-clockwise is parallel to $\ell$. If the orientation of a line or line segment is $\theta$, then we say that the line or line segment is $\theta$-aligned.

A $\theta$-aligned rectangle (or square) for $\theta \in[0, \pi / 2)$ is a rectangle (square, resp.) whose sides are either $\theta$-aligned or $(\theta+\pi / 2)$-aligned. Note that any rectangle or square in the plane is $\theta$-aligned for a unique $\theta \in[0, \pi / 2)$. Two rectangles or squares are said to be parallel if both are $\theta$-aligned for some $\theta \in[0, \pi / 2)$. For a square, its center is the intersection point of its two diagonals and its radius is half its side length. Two squares are called concentric if they are parallel and share a common center. We denote by area $(R)$ the area of a rectangle or square $R$.

In each orientation $\theta \in[0, \pi / 2)$, we regard any $\theta$-aligned line to be horizontal and directed in the $x$-coordinate increasing direction, while any $(\theta+\pi / 2)$-aligned line to be vertical and directed in the $y$-coordinate increasing direction. For any $p, q \in \mathbb{R}^{2}$, we say that $p$ is to the left of $q$, or $q$ is to the right of $p$, in orientation $\theta$ if the orthogonal projection of $p$ onto a $\theta$-aligned (directed) line is prior to that of $q$. Analogously, $p$ is below $q$, or equivalently, $q$ is above $p$ in $\theta$ if the orthogonal projection of $p$ onto a $(\theta+\pi / 2)$-aligned (directed) line is prior to that of $q$. This also enables us to identify the top, bottom, left, and right sides of any rectangle or square in any orientation $\theta \in[0, \pi / 2)$.

Square and rectangular annuli. A square annulus $A$ is defined to be the closed region between two concentric squares, called the outer and inner squares of $A$. Its width $\operatorname{width}(A)$ is the difference of the radii of its outer and inner squares, and its area area $(A)$ is the difference of the areas of its outer and inner squares. See Figure 1(a) for an illustration.

A rectangular annulus $A$ is the closed region between two parallel rectangles $R$ and $R^{\prime}$ with $R^{\prime} \subseteq R$, that is, $A=R \backslash \operatorname{int} R^{\prime}$. The two rectangles $R$ and $R^{\prime}$ defining $A$ as above are called the outer and inner rectangles of $A$, respectively. The perpendicular distance between the top side of $R$ and the top side of $R^{\prime}$ is called the top-width of $A$. Analogously, we define the bottom-width, left-width, and right-width of rectangular annulus $A$. The width width $(A)$ of $A$ is defined to be the maximum of its top-, bottom-, left-, and right-widths. If the top-, bottom-, left-, and right-widths of $A$ are all equal, then $A$ is called a uniform-width (or, shortly uniform) rectangular annulus. The area area $(A)$ of $A$ is simply the difference area $(R)-\operatorname{area}\left(R^{\prime}\right)$ of the areas of its outer and inner rectangles. See Figure $1(b-c)$ for an illustration.

A rectangular or square annulus is also called $\theta$-aligned if its outer and inner rectangles or squares are $\theta$-aligned. If an annulus is 0 -aligned, then it is rather called axis-parallel. 
Problem definition. In this paper, we study the problem of computing a rectangular or square annulus of minimum extent that encloses a given set $P$ of $n$ points in the plane either in a fixed orientation or over all orientations $\theta \in[0, \pi / 2)$. The objective extents to minimize we are interested in are width, area, or their combinations.

To be more precise, for each $\theta \in[0, \pi / 2)$, let $\mathcal{S}(\theta), \mathcal{R}(\theta)$, and $\mathcal{R}_{u}(\theta)$ be the set of all $\theta$-alignes square, rectangular, and uniform rectangular annuli, respectively, that enclose $P$. By definition, note that $\mathcal{S}(\theta) \subset \mathcal{R}_{u}(\theta) \subset \mathcal{R}(\theta)$. Also, let $\mathcal{S}:=\bigcup_{\theta \in[0, \pi / 2)} \mathcal{S}(\theta), \mathcal{R}:=\bigcup_{\theta \in[0, \pi / 2)} \mathcal{R}(\theta)$, and $\mathcal{R}_{u}:=\bigcup_{\theta \in[0, \pi / 2)} \mathcal{R}_{u}(\theta)$. For each class $\mathcal{A} \in\left\{\mathcal{S}, \mathcal{R}, \mathcal{R}_{u}\right\}$, we consider the following optimization problems:

- The minimum-width problem asks to minimize width $(A)$ over all $A \in \mathcal{A}(\theta)$ for a fixed orientation $\theta \in[0, \pi / 2)$, or over all $A \in \mathcal{A}$ for arbitrary orientation.

- The minimum-area problem asks to minimize area $(A)$ over all $A \in \mathcal{A}(\theta)$ for a fixed orientation $\theta \in[0, \pi / 2)$, or over all $A \in \mathcal{A}$ for arbitrary orientation.

In most cases, there may be two or more annuli of a particular shape with minimum width or minimum area. This yields a series of bicriteria optimization problems. Here, in this paper, two variants are discussed.

- The minimum-area minimum-width problem asks to find an optimal annulus whose area is the smallest among those with minimum width, either in a fixed orientation or over all orientations.

- The minimum-width minimum-area problem asks to find an optimal annulus whose width is the smallest among those with minimum area, either in a fixed orientation or over all orientations.

\section{Annuli in Fixed Orientation}

In this section, we study the problem of computing a minimum-area square and rectangular annulus in a given orientation $\theta \in[0, \pi / 2)$, and its variations. Without loss of generality, we assume $\theta=0$, so our annuli in mind are all axis-parallel. We first handle the square case and move on to the rectangular case.

\subsection{Axis-parallel square annulus}

Let $P$ be a set of $n$ input points in the plane $\mathbb{R}^{2}$. We show that any minimum-area axis-parallel square annulus is indeed a minimum-width axis-parallel square annulus. For the purpose, we review a necessary observation about minimum-width square annuli.

Lemma 1 (Gluchshenko et al. [15]) There exists a minimum-width square annulus enclosing $P$ such that its outer square is a smallest enclosing square for $P$, that is, a pair of its opposite sides contain a point of $P$ on each.

For the minimum-area case, we observe the following.

Lemma 2 Let $A$ be any minimum-area axis-parallel square annulus enclosing $P$. Then, the outer square of $A$ is a smallest enclosing axis-parallel square for $P$.

Proof. Suppose for the contradiction that the outer square $S$ of $A$ is not a smallest enclosing square for $P$. Let $A^{\prime}$ be a minimum-width square annulus such that whose outer square $S^{\prime}$ is a smallest enclosing square for $P$. Such an annulus $A^{\prime}$ exists by Lemma 1 . We then have width $(A) \geq \operatorname{width}\left(A^{\prime}\right)$ and $\operatorname{area}(S)>\operatorname{area}\left(S^{\prime}\right)$. This implies that area $(A)>\operatorname{area}\left(A^{\prime}\right)$, a contradiction. 
Lemma 2 indeed fixes the size of the outer square of any possible minimum-area square annulus, and thus the problem is reduced to finding such a minimum-width annulus described in Lemma 1.

Lemma 3 Let $A$ be an axis-parallel square annulus enclosing $P$. Then, $A$ is of the minimumarea if and only if $A$ is of the minimum-width and its outer square is a smallest enclosing square for $P$.

The algorithm by Gluchshenko et al. [15] indeed computes a minimum-width square annulus whose outer square is a smallest enclosing square for $P$ based on Lemma1, and they also proved a lower bound $\Omega(n \log n)$ for the minimum-width problem. Hence, we conclude the following.

Theorem 1 A minimum-area square annulus in a fixed orientation enclosing $n$ points can be computed in optimal $O(n \log n)$ time.

Note that the algorithm by Gluchshenko et al. can be easily modified to find all minimumwidth square annuli whose outer square is a smallest enclosing square for $P$ in $O(n \log n)$ time. Thus, a minimum-area minimum-width or minimum-width minimum-area square annulus in a fixed orientation can be computed in the same time bound, and this is time-optimal.

Corollary 1 A minimum-area minimum-width and a minimum-width minimum-area square annulus in a fixed orientation can be computed in optimal $O(n \log n)$ time.

\subsection{Axis-parallel rectangular annuli}

Next, we consider axis-parallel rectangular annuli. The following is a well-known observation on the minimum-width rectangular annuli.

Lemma 4 (Abellanas et al. [1] and Mukherjee et al. 18]) There exists a minimum-width axisparallel rectangular annulus enclosing $P$ such that its outer rectangle is the smallest enclosing axis-parallel rectangle for $P$. The same holds for a minimum-width axis-parallel uniform rectangular annulus.

It is not difficult to see that for the minimum-width problem, rectangular annuli and uniform rectangular annuli are equivalent. Here, we give a direct and simple proof for the fact.

Lemma 5 Let $A$ be a minimum-width uniform rectangular annulus enclosing $P$. Then, $A$ is also a minimum-width rectangular annulus enclosing $P$.

Proof. Let $A^{\prime}$ be a minimum-width rectangular annulus enclosing $P$. Since any uniform rectangular annulus is also a rectangular annulus, it holds that $\operatorname{width}\left(A^{\prime}\right) \leq \operatorname{width}(A)$ in general. Suppose to the contrary that $\operatorname{width}\left(A^{\prime}\right)<\operatorname{width}(A)$, and assume without loss of generality that the width width $\left(A^{\prime}\right)$ of $A^{\prime}$ is determined by the top-width of $A^{\prime}$. Then, by sliding each side of the inner rectangle of $A^{\prime}$ inwards, one can obtain a new uniform rectangular annulus $A^{\prime \prime}$ with $\operatorname{width}\left(A^{\prime \prime}\right)=\operatorname{width}\left(A^{\prime}\right)<\operatorname{width}(A)$, a contradiction.

Unlike the minimum-width problem, the minimum-area problem behaves differently for uniform and general rectangular annuli. Our first observation on the minimum-area problem for rectangular annuli is about their outer rectangles.

Lemma 6 Let $A$ be a minimum-area axis-parallel rectangular annulus or a minimum-area uniform axis-parallel rectangular annulus enclosing $P$. In either case, its outer rectangle should be the smallest enclosing axis-parallel rectangle for $P$.

Proof. Suppose that the outer rectangle $R$ of $A$ is not the smallest enclosing rectangle for $P$. Then, replacing $R$ by the smallest enclosing rectangle for $P$ results in an annulus enclosing $P$ with smaller area, a contradiction.

This already implies the following. 
Theorem 2 A minimum-area uniform rectangular annulus enclosing $P$ in a fixed orientation is unique and it coincides with the minimum-width uniform rectangular annulus whose outer rectangle is the smallest enclosing rectangle for $P$ in the orientation. Therefore, it can be computed in $O(n)$ time.

Proof. By Lemma 6, the outer rectangle of an axis-parallel minimum-area uniform rectangular annulus enclosing $P$ should be the smallest axis-parallel rectangle $R$ enclosing $P$. For a fixed outer rectangle $R$, minimizing the area is equivalent to minimizing the width over all uniform rectangular annuli enclosing $P$. Hence, the axis-parallel minimum-area uniform rectangular annulus enclosing $P$ is uniquely determined by the minimum-width axis-parallel uniform rectangular annulus whose outer rectangle is $R$. Such an annulus exists by Lemmas 4 and 5 , and can easily computed in $O(n)$ time by computing the distance from each point in $P$ to the boundary of the outer rectangle $R$, as done in Abellanas [1].

By the uniqueness and the characterization of Theorem 2, the minimum-area minimumwidth and minimum-width minimum-area uniform rectangular annulus in a fixed orientation can also be computed in $O(n)$ time.

Corollary 2 A minimum-area minimum-width or minimum-width minimum-area uniform rectangular annulus enclosing $P$ in a fixed orientation can be computed in $O(n)$ time.

We then turn to the case of general rectangular annuli. For the general rectangular annulus, Lemma 6 also fixes the outer rectangle as the smallest enclosing rectangle $R$ for $P$. Hence, the minimum-area problem is now reduced to maximizing the area of the inner rectangle. Let $R^{\prime}$ be a possible inner rectangle that maximizes its area. Then, $R^{\prime}$ must satisfy the following conditions: (1) $R^{\prime}$ is empty of points in $P$, that is, no point in $P$ lies in the interior of $R^{\prime}$. (2) $R^{\prime}$ is contained in $R$, that is, $R^{\prime} \subseteq R$. Such a rectangle $R^{\prime}$ satisfying conditions (1) and (2) is called an empty rectangle among $P$. This directly shows a reduction to the problem of finding an axis-parallel largest empty rectangle among points $P$. The largest empty rectangle problem takes an axis-parallel rectangle $B$ and a set $Q$ of $n$ points in $B$ as input, and asks to find a maximum-area axis-parallel rectangle that is empty of $P$ and is contained in $B$.

Lemma 7 The problem of computing a minimum-area axis-parallel rectangular annulus enclosing $P$ is computationally equivalent to the problem of computing a largest empty axis-parallel rectangle among $P$. More precisely, both problems are reduced to each other in linear time.

Proof. Let $P$ be an instance of the minimum-area axis-parallel rectangular annulus problem. As discussed above, Lemma 6 tells us the outer rectangle of the solution should be the smallest enclosing rectangle $R$ for $P$. Then, we can find the inner rectangle of maximum area by solving the largest empty rectangle problem for input $R$ and $P$. This shows a reduction to the largest empty rectangle problem in $O(n)$ time.

Next, consider an input $(B, Q)$ of the largest empty rectangle problem, where $Q$ is a set of $n$ points in rectangle $B$. To solve the problem, we apply any algorithm for the minimum-area rectangular annulus problem for input $P:=Q \cup\left\{p_{1}, p_{2}\right\}$, where $p_{1}$ is the top-left corner and $p_{2}$ is the bottom-right corner of $B$, and let $A$ be the output optimal annulus. By Lemma 6 , the outer rectangle of $A$ is $B$. On the other hand, the inner rectangle $R^{\prime}$ of $A$ should maximize its area while $R^{\prime}$ is empty of $P$ and $R^{\prime} \subseteq B$. Such a rectangle $R^{\prime}$ is of course a largest empty rectangle. This completes a reduction to the minimum-area rectangular annulus problem in $O(n)$ time.

Hence, our problem can be solved by applying any algorithm for the largest empty rectangle problem. The largest empty rectangle problem is one of the classical and well-studied problems in computational geometry. There are two approaches to solve the problem: whether one checks all maximal empty rectangles or not. Given a set $P$ of points and its bounding rectangle $R$, a (axis-parallel) maximal empty rectangle is an empty rectangle each of whose edges either 
contains a point in $P$ or a portion of an edge of $R$. Naamad et al. 19 presented an algorithm for the largest empty rectangle problem that runs in $O\left(\min \left\{r \log n, n^{2}\right\}\right)$ time, where $r$ denotes the number of maximal empty rectangles. In the same paper, it is also shown that $r=O\left(n^{2}\right)$ in the worst case and $r=O(n \log n)$ in expectation. This algorithm was improved by Orlowski 20 to $O(n \log n+r)$ time. Chazelle et al. [12] presented an $O\left(n \log ^{3} n\right)$-time algorithm that does not enumerate all maximal empty rectangles, and Aggarwal and Suri [5] improved it to $O\left(n \log ^{2} n\right)$ time with $O(n)$ space. Mckenna et al. [17] proved a lower bound of $\Omega(n \log n)$ for this problem.

Theorem 3 A minimum-area rectangular annulus enclosing $P$ in a fixed orientation can be computed in $O\left(n \log ^{2} n\right)$ or $O(n \log n+r)$ time in the worst case, or in $O(n \log n)$ expected time, where $r=O\left(n^{2}\right)$ denotes the number of maximal empty rectangles among $P$. Moreover, any algorithm for the problem takes $\Omega(n \log n)$ time in the worst case.

Proof. The first statement directly follows from Lemma 7 and the above discussion. The currently fastest algorithms for the largest empty rectangle problem are one by Aggarwal and Suri [5] with running time $O\left(n \log ^{2} n\right)$ and the other by Orlowski [20] with running time $O(n \log n+r)$. As proved by Namaad et al. [19], $r=O\left(n^{2}\right)$ in the worst case and $r=O(n \log n)$ in expectation, so the algorithm by Orlowski runs in $O(n \log n)$ expected time. Finally, again by Lemma 7, the lower bound $\Omega(n \log n)$ by Mckenna et al. [17] applies to the minimum-area rectangular annulus problem in a fixed orientation.

A minimum-area minimum-width rectangular annulus can be found as follows: First, compute the minimum-width uniform rectangular annulus $A_{0}$ in $O(n)$ time, and let $w$ be its width and $R_{0}^{\prime}$ be its inner rectangle. We need to find a maximum-area empty rectangle $R^{\prime}$ that contains $R_{0}^{\prime}$ in order to keep the width of the annulus formed by $R$ and $R^{\prime}$ at most $w$, that is, being the minimum width. Since $A_{0}$ is a minimum-width annulus, we further observe that at least one side of $R^{\prime}$ must be overlapped with a side of $R_{0}^{\prime}$. Without loss of generality, we assume that the bottom side of $R^{\prime}$ is a segment on the line $\ell$ supporting the bottom side of $R_{0}^{\prime}$. Then, $R^{\prime}$ can be found by enumerating all maximal empty rectangles whose bottom side lies on $\ell$, testing if each contains $R_{0}^{\prime}$, and, if so, checking its area. Fortunately, Orlowski [20, Section 2] showed how to enumerate those maximal empty rectangles whose bottom side lies on $\ell$ in $O(n)$ time, after sorting the points in $P$ in their $x$ - and $y$-coordinates. Thus, we conclude the following.

Theorem 4 A minimum-area minimum-width rectangular annulus enclosing $P$ in a fixed orientation can be computed in $O(n \log n)$ time.

A minimum-width minimum-area rectangular annulus can be found by checking all largest empty rectangles for $P$. However, known algorithms [5, 12] that do not enumerate all maximal empty rectangles do not guarantee to find all largest empty rectangles. Hence, we apply the algorithm by Orlowski [20] and check every maximal empty rectangle and the width of the corresponding annulus. This takes $O(n \log n+r)$ time.

Theorem 5 A minimum-width minimum-area rectangular annulus enclosing $P$ in a fixed orientation can be computed in $O(n \log n+r)$ time, where $r$ denotes the number of maximal empty rectangles among $P$.

\section{Rectangular Annuli in Arbitrary Orientation}

In this section, we consider the problem of computing an optimal rectangular annulus in arbitrary orientation for different objectives.

We will often discuss the distance between the orthogonal projections of $p$ and $q$ onto a $\theta$-aligned line for $\theta \in[0, \pi)$, denoted by $w_{\theta}(p, q)$. It is not difficult to see that

$$
w_{\theta}(p, q)=|\overline{p q}| \cdot\left|\cos \left(\theta_{p q}-\theta\right)\right|,
$$




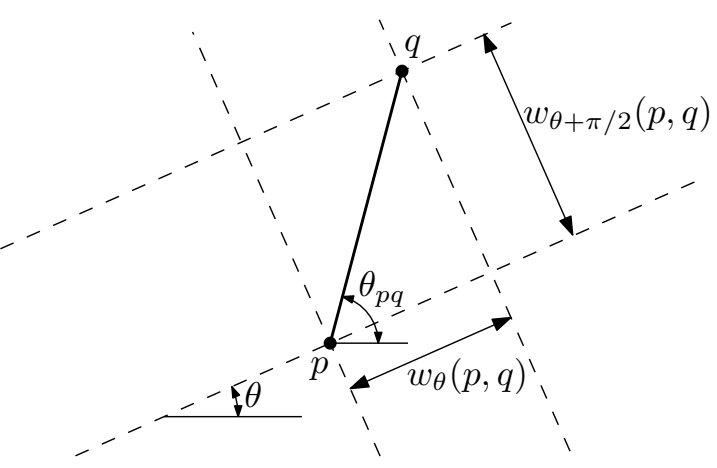

(a)

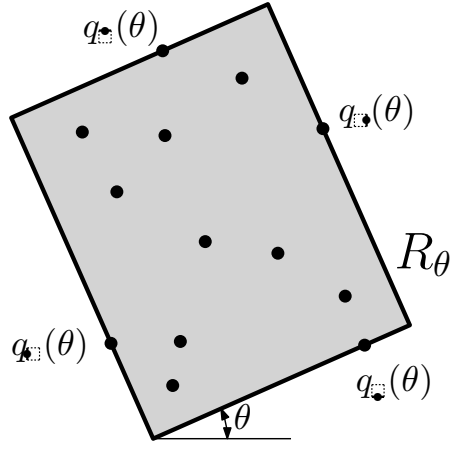

(b)

Figure 2: For $\theta \in[0, \pi / 2)$, (a) $w_{\theta}(p, q)$ and $w_{\theta+\pi / 2}(p, q)$ for two points $p, q \in \mathbb{R}^{2}$, and (b) the smallest enclosing $\theta$-aligned rectangle $R_{\theta}$ for points $P$.

where $\theta_{p q} \in[0, \pi)$ denotes the orientation of $\overline{p q}$. Intuitively, one may regard that $w_{\theta}(p, q)$ is the horizontal distance and $w_{\theta+\pi / 2}(p, q)$ is the vertical distance between $p$ and $q$ in orientation $\theta \in[0, \pi / 2)$. See Figure 2(a).

As the side length of a $\theta$-aligned rectangle or square of our interests is often described by a combination of $w_{\theta}$ with different pairs of points $(p, q)$, we first introduce some basic properties of such functions of variable $\theta$. A function of a particular form $a \sin (\omega \theta+\phi)+b$ for some constants $a, b, \omega, \phi \in \mathbb{R}$ is called a sinusoidal function, where $a, b, \omega$ and $\phi$ are called its amplitude, bas $\epsilon^{1}$, (angular) frequency and phase, respectively. Note that $\cos (\theta)$ is also sinusoidal with base 0 as $\cos (\theta)=\sin (\theta+\pi / 2)$. Observe that for a fixed pair of points $p, q \in \mathbb{R}^{2}, w_{\theta}(p, q)$ is a piecewise sinusoidal function of base 0 and frequency 1 over $\theta \in[0, \pi)$ with at most one breakpoint at $\theta=\theta_{p q}-\pi / 2$ or $\pi / 2-\theta_{p q}$.

The following properties of sinusoidal functions are well known and easily derived.

Observation 1 The sum of two sinusoidal functions of base 0 and frequency $\omega$ is also sinusoidal of base 0 and frequency $\omega$. Therefore, the graphs of two sinusoidal functions of equal frequency cross at most once in domain $[0, \pi)$.

Observation 2 The product of two sinusoidal functions of base 0 and frequency $\omega$ is equal to a sinusoidal functions of frequency $2 \omega$. Specifically, it holds that

$$
a_{1} \sin \left(\omega \theta+\phi_{1}\right) \cdot a_{2} \sin \left(\omega \theta+\phi_{2}\right)=\frac{a_{1} a_{2}}{2}\left(\sin \left(2 \omega \theta+\phi_{1}+\phi_{2}-\frac{\pi}{2}\right)+\cos \left(\phi_{1}-\phi_{2}\right)\right),
$$

for any constants $a_{1}, a_{2}, \phi_{1}, \phi_{2}, \omega \in \mathbb{R}$.

\subsection{Uniform rectangular annuli}

For any orientation $\theta \in[0, \pi / 2)$, let $R_{\theta}$ be the smallest $\theta$-aligned rectangle enclosing $P$. The rectangle $R_{\theta}$ is determined by the leftmost, rightmost, topmost, and bottommost points in orientation $\theta$ among those in $P$, in such a way that each side of $R_{\theta}$ contains each of these four points. Let $q_{*}(\theta), q_{*}(\theta), q_{*}(\theta), q_{*}(\theta) \in P$ be the topmost, bottommost, leftmost, and rightmost points in orientation $\theta$ among those in $P$. (If there are two or more topmost, bottommost, leftmost, or rightmost points in $\theta$, then we choose an arbitrary one.) See Figure 2(b). For each $p \in P$ and $\theta \in[0, \pi / 2)$, we define a function $f_{p}:[0, \pi / 2) \rightarrow \mathbb{R}$ to be the "distance" from the

\footnotetext{
${ }^{1}$ The constant $b$ is usually called the center amplitude in wave analysis, while here we call it base for simplicity.
} 
boundary of $R_{\theta}$. More precisely, $f_{p}(\theta)$ takes the minimum among the perpendicular distances from every side of $R_{\theta}$ to $p$ :

$$
f_{p}(\theta):=\min \left\{w_{\theta+\pi / 2}\left(p, q_{\bullet}(\theta)\right), w_{\theta+\pi / 2}\left(p, q_{*}(\theta)\right), w_{\theta}\left(p, q_{*}(\theta)\right), w_{\theta}\left(p, q_{*}(\theta)\right)\right\} .
$$

Now, we let $w(\theta)$ be the width of a minimum-width $\theta$-aligned rectangular annulus enclosing $P$. By Lemma 4, there exists a minimum-width uniform $\theta$-aligned rectangular annulus whose outer rectangle is $R_{\theta}$. Note that such a uniform rectangular annulus is unique and we denote it by $A_{u}(\theta)$. Moreover, Lemma 5 states that $A_{u}(\theta)$ is also a minimum-width $\theta$-aligned rectangular annulus. Hence, we have

$$
w(\theta)=\operatorname{width}\left(A_{u}(\theta)\right)=\max _{p \in P} f_{p}(\theta) .
$$

The above discussion already results in an $O\left(n^{2} \log n\right)$-time algorithm for the minimumwidth rectangular annulus problem over all orientations $\theta \in[0, \pi / 2)$. The minimum-width problem can be solved by computing $\min _{\theta \in[0, \pi / 2)} w(\theta)$ and the corresponding annulus. Observe that for an interval $I \subset[0, \pi / 2)$ such that the tuple of four points $\left(q_{*}(\theta), q_{*}(\theta), q_{\mathfrak{*}}(\theta), q_{*}(\theta)\right)$ is fixed, each function $f_{p}$ restricted to $I$ is piecewise sinusoidal with at most eight pieces. In such an interval $I$, minimizing $w$ over $\theta \in I$ can be done by computing the upper envelope of functions $f_{p}$ and finding a lowest point in the envelope.

Lemma 8 Let $I \subset[0, \pi / 2)$ be an interval in which the four points $q_{*}(\theta), q_{*}(\theta), q_{*}(\theta), q_{*}(\theta)$ determining $R_{\theta}$ are fixed. Then, the function $w$ over $I$ is a piecewise frequency-1 sinusoidal function of complexity $O(n \alpha(n))$ and can be explicitly computed in $O(n \log n)$ time.

Proof. Note that function $w$ is the upper envelope of $n$ functions $f_{p}$ for $p \in P$. Since $f_{p}$ over $I$ consists of at most eight sinusoidal curves for each $p \in P$, function $w$ over $I$ is the upper envelope of $O(n)$ curves that are sinusoidal of frequency 1. By Observation 1, any two of these curves cross at most once. Thus, the upper envelope $w$ on $I$ can be explicitly computed in $O(n \log n)$ time [16, while $w$ consists of $O(n \alpha(n))$ sinusoidal pieces [22].

Finally, as observed earlier by Toussaint [23] and also applied by Mukherjee et al. [18] and Bae [7], the domain $[0, \pi / 2)$ is decomposed into at most $O(n)$ such intervals $I$.

Lemma 9 (Toussaint [23]) There are at most $O(n)$ changes in the tuple of four points $\left(q_{*}(\theta)\right.$, $\left.q_{*}(\theta), q_{*}(\theta), q_{3}(\theta)\right)$ as a function of $\theta \in[0, \pi / 2)$. That is, the domain $[0, \pi / 2)$ is partitioned into $O(n)$ maximal intervals, in each of which the four points stay constant.

Each such interval described in Lemma 9 is called primary. Therefore, in $O\left(n^{2} \log n\right)$ time, we can compute a minimum-width rectangular annulus enclosing $P$ over all orientation ${ }^{2}$.

Note that the above algorithm indeed finds all minimum-width uniform rectangular annulus enclosing $n$ points over all orientations. Hence, by picking one with minimum area among all of them, we can find a minimum-area minimum-width uniform rectangular annulus.

Theorem 6 A minimum-area minimum-width uniform rectangular annulus enclosing $n$ points over all orientations can be computed in $O\left(n^{2} \log n\right)$ time.

Now, we turn to the minimum-area problem for uniform rectangular annuli. Recall Theorem 2 stating that $A_{u}(\theta)$ is indeed the unique minimum-area $\theta$-aligned uniform rectangular annulus enclosing $P$. So, we want to minimize area $\left(A_{u}(\theta)\right)$ over $\theta \in[0, \pi / 2)$. The area area $\left(A_{u}(\theta)\right)$ can be represented as follows:

$$
\begin{aligned}
\operatorname{area}\left(A_{u}(\theta)\right) & =\operatorname{peri}\left(R_{\theta}\right) \cdot w(\theta)-4(w(\theta))^{2} \\
& =2\left(w_{\theta+\pi / 2}\left(q_{\bullet}(\theta), q_{\bullet}(\theta)\right)+w_{\theta}\left(q_{\bullet}(\theta), q_{\bullet}(\theta)\right)\right) \cdot w(\theta)-4(w(\theta))^{2},
\end{aligned}
$$

\footnotetext{
${ }^{2}$ The $O\left(n^{2} \log n\right)$-time algorithm presented by Mukherjee et al. 18 is slightly different, but its outline and approach is almost identical to that we describe here.
} 
where peri $(\cdot)$ denotes the perimeter of a rectangle.

Consider any primary interval $I \subset[0, \pi / 2)$. In $I$, the four extreme points $q_{\bullet}=q_{*}(\theta)$, $q_{*}=q_{*}(\theta), q_{*}=q_{\boldsymbol{*}}(\theta)$, and $q_{\boldsymbol{*}}=q_{*}(\theta)$ are fixed, so each of the terms $w_{\theta+\pi / 2}\left(q_{*}, q_{*}\right)$ and $w_{\theta}\left(q_{*}, q_{*}\right)$ are piecewise base- 0 frequency-1 sinusoidal, and so is their sum by Observation 1 . Further, Lemma 8 tells us that $w(\theta)$ is also piecewise base- 0 frequency- 1 sinusoidal over $I$. From the above discussion, we then observe that area $\left(A_{u}(\theta)\right)$ is piecesise frequency-2 sinusoidal by Observation 2. Hence, we can compute an explicit description of the function area $\left(A_{u}(\theta)\right)$ over $\theta \in I$, provided that the description of function $w$ has been computed, in time proportional to the number of breakpoints of $w$.

Theorem 7 A minimum-area uniform rectangular annulus enclosing $n$ points over all orientations can be computed in $O\left(n^{2} \log n\right)$ time.

Proof. We handle each primary interval $I \subset[0, \pi / 2)$ in which the four extreme points $q_{\bullet}=$ $q_{*}(\theta), q_{*}=q_{*}(\theta), q_{*}=q_{*}(\theta)$, and $q_{*}=q_{*}(\theta)$ are fixed. Apply Lemma 8 to explicitly compute function $w$ on $I$ in $O(n \log n)$ time. Note that $w$ is a piecewise sinusoidal function of $O(n \alpha(n))$ breakpoints. As briefly discussed above, the term $w_{\theta+\pi / 2}\left(q_{\bullet}, q_{*}\right)+w_{\theta}\left(q_{*}, q_{*}\right)=\operatorname{peri}\left(R_{\theta}\right) / 2$ is a piecewise sinusoidal function of $O(1)$ breakpoints. Compute a sorted list of all breakpoints of $w$ and peri $\left(R_{\theta}\right)$ in $O(n \alpha(n))$ time. This is possible because the breakpoints of $w$ are already given sorted from Lemma 8 . We then compute the function area $\left(A_{u}(\theta)\right)$ between every two consecutive breakpoints and find a minimum in there. Note that area $\left(A_{u}(\theta)\right)$ between two consecutive breakpoints is a sinusoidal function of frequency 2 by Observation 2 and can be minimized in $O(1)$ time.

Consequently, we can minimize area $\left(A_{u}(\theta)\right)$ over $\theta \in I$ in $O(n \log n)$ time. Since there are $O(n)$ primary intervals by Lemma 9, our algorithm takes $O\left(n^{2} \log n\right)$ time in total.

Remark that our algorithm described in Theorem 7 indeed finds all minimum-area uniform rectangular annulus enclosing $n$ points over all orientations. Hence, by selecting one with minimum width among all of them, we can find a minimum-width minimum-area uniform rectangular annulus.

Corollary 3 A minimum-width minimum-area uniform rectangular annulus enclosing $n$ points over all orientations can be computed in $O\left(n^{2} \log n\right)$ time.

\subsection{Minimum-area minimum-width rectangular annulus}

Next, we solve the minimum-area minimum-width rectangular annulus problem. For the purpose, we specify all orientations in which the width function $w(\theta)$ is minimized over $\theta \in[0, \pi / 2)$, and then we find a minimum-area rectangular annulus in each of these orientations, applying the algorithm of Theorem 4.

Theorem 8 A minimum-area minimum-width rectangular annulus enclosing $n$ points over all orientations can be computed in $O\left(n^{2} \log n+t n\right)$ time, where $t=O\left(n^{2}\right)$ denotes the number of different orientations $\theta^{\prime}$ such that $w\left(\theta^{\prime}\right)=\min _{\theta \in[0, \pi / 2)} w(\theta)$.

Proof. As described above, we first compute the explicit description of function $w$ over $\theta \in$ $[0, \pi / 2)$ in $O\left(n^{2} \log n\right)$ time as described in the previous section by Lemmas 8 and 9 . Since we identify the full description of $w$, we can specify all orientations that minimize $w$. Let $\Theta$ be the set of all orientations $\theta \in[0, \pi / 2)$ that minimize $w$ in the same time bound. Note that the orientations in $\Theta$ are obtained in the sorted order. We then apply the algorithm of Theorem 4 for each $\theta \in \Theta$. Provided the sorted lists of $P$ in orientation $\theta$ and $\theta+\pi / 2$, this takes $O(n)$ time for each $\theta \in \Theta$. As $\theta$ continuously increases from 0 to $\pi / 2$, the sorted lists of $P$ in orientation $\theta$ and $\theta+\pi / 2$ can be maintained in total $O\left(n^{2} \log n\right)$ time after sorting the orientations of all lines through two points in $P$ in $O\left(n^{2} \log n\right)$ time. Thus, a minimum-area minimum-width 
rectangular annulus enclosing $P$ can be found in $O\left(n^{2} \log n+t n\right)$ time, where $t=|\Theta|$.

Now, we bound the cardinality $t$ of $\Theta$. As proved in Lemma 8 , the complexity of $w$ in a primary interval $I$ is $O(n \alpha(n))$. This already implies that $t=O\left(n^{2} \alpha(n)\right)$ by Lemma 9. Here, we prove that $t=O\left(n^{2}\right)$ in fact.

Let $w^{*}:=\min _{\theta \in[0, \pi / 2)} w(\theta)$ be the minimum width over $\theta \in[0, \pi / 2)$. In each primary interval $I \subset[0, \pi / 2)$, recall that the width function $w$ is the upper envelope of $n$ functions $f_{p}$ and $f_{p}$ is a piecewise frequency- 1 sinusoidal function of $O(1)$ breakpoints. By Observation 1 . for each $p \in P$, there are $O(1)$ different orientations $\theta \in I$ such that $f_{p}(\theta)=w^{*}$. Hence, we have $|\Theta \cap I|=O(n)$ for any primary interval $I \subset[0, \pi / 2)$, and $t=|\Theta|=O\left(n^{2}\right)$ by Lemma 9 , $\square$

\subsection{Minimum-area rectangular annulus}

Finally, we consider the minimum-area problem for general rectangular annuli in arbitrary orientation. For a fixed orientation $\theta \in[0, \pi / 2)$, recall that the problem is equivalent to the largest empty rectangle problem in orientation $\theta$ as shown in Lemma 7, so we can find a largest empty $\theta$-aligned rectangle in the smallest enclosing $\theta$-aligned rectangle $R_{\theta}$ for $P$.

Let $a(\theta)$ be the area of a minimum-area $\theta$-aligned rectangular annulus enclosing $P$. Our problem here is to minimize $a(\theta)$ over $\theta \in[0, \pi / 2)$ and to find the corresponding annulus. By Lemma 7, the inner rectangle of an optimal annulus over all orientations is a maximal empty rectangle among $P$ in some orientation. A maximal empty rectangle among $P$ in arbitrary orientation is defined to be a $\theta$-aligned empty rectangle $E_{\theta}$ for $\theta \in[0, \pi / 2)$ such that $E_{\theta}$ is contained in $R_{\theta}$ and there is no other $\theta$-aligned empty rectangle $E_{\theta}^{\prime}$ with $E_{\theta} \subset E_{\theta}^{\prime}$. This implies that each side of any maximal empty rectangle either contains a point of $P$ or is a portion of a side of $R_{\theta}$. We then represent a maximal empty rectangle $E_{\theta}$ by a tuple $\left(p_{\bullet}, p_{\bullet}, p_{\bullet}, p_{*} ; \theta\right)$, where $\theta$ is the orientation of $E_{\theta}$, and

- $p_{\bullet}$ denotes a point in $P$ lying on the top side of $E$, if any, or $p_{\bullet}=q \cdot(\theta)$, otherwise;

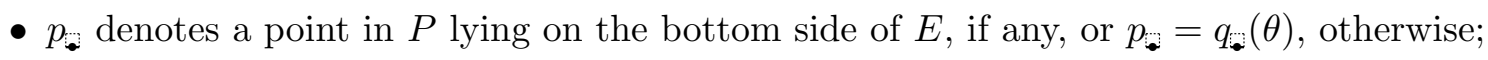

- $p_{\bullet}$ denotes a point in $P$ lying on the left side of $E$, if any, or $p_{E}=q_{E}(\theta)$, otherwise;

- $p_{\text {a }}$, denotes a point in $P$ lying on the right side of $E$, if any, or $p_{:}=q_{*}(\theta)$, otherwise.

Two maximal empty rectangles $E_{\theta}=\left(p_{*}, p_{*}, p_{\bullet}, p_{*} ; \theta\right)$ and $E_{\theta^{\prime}}^{\prime}=\left(p_{\bullet}^{\prime}, p_{*}^{\prime}, p_{*}^{\prime}, p_{;}^{\prime} ; \theta^{\prime}\right)$ with $\theta \leq \theta^{\prime}$ are said to be combinatorially equivalent if $p_{\ldots}=p^{\prime}$ for each $\in\{\bullet, \infty, \infty\}$ and all rectangles represented by $\left(p_{\bullet}, p_{\bullet}, p_{\bullet}, p_{*} ; \theta^{\prime \prime}\right)$ for $\theta \leq \theta^{\prime \prime} \leq \theta^{\prime}$ are maximal empty rectangles among $P$. We call a maximal set of combinatorially equivalent maximal empty rectangles among $P$ a MER class. A MER class is thus represented by a tuple $\left(p_{\bullet}, p_{\bullet}, p_{\bullet}, p_{*} ; J\right)$, where $J \subseteq[0, \pi / 2)$ is an orientation interval such that $\left(p_{\bullet}, p_{\bullet}, p_{\bullet}, p_{;} ; \theta\right)$ is a maximal empty rectangle for each $\theta \in J$. We shall call $J$ the valid interval of MER class $E$. Chaudhuri et al. 11 proved that there are $O\left(n^{3}\right)$ different MER classes, and presented an $O\left(n^{3}\right)$-time algorithm to specify all of them.

Let $E=\left(p_{\bullet}, p_{\bullet}, p_{\bullet}, p_{*} ; J\right)$ be a MER class, and $E_{\theta}:=\left(p_{\bullet}, p_{*}, p_{\bullet}, p_{*} ; \theta\right)$ for $\theta \in J$ be the maximal empty $\theta$-aligned rectangle in $E$. Then, the area area $\left(E_{\theta}\right)$ of $E_{\theta}$ is

$$
\operatorname{area}\left(E_{\theta}\right)=w_{\theta+\pi / 2}\left(p_{\bullet}, p_{\bullet}\right) \cdot w_{\theta}\left(p_{\bullet}, p_{\bullet}\right),
$$

which is the product of two sinusoidal functions of base 0 and frequency 1 . Define $a_{E}(\theta)$ be the area of the rectangular annulus whose outer rectangle is $R_{\theta}$ and inner rectangle is $E_{\theta}$. We then have

$$
\begin{aligned}
a_{E}(\theta) & =\operatorname{area}\left(R_{\theta}\right)-\operatorname{area}\left(E_{\theta}\right) \\
& =w_{\theta+\pi / 2}\left(q_{\bullet}(\theta), q_{\bullet}(\theta)\right) \cdot w_{\theta}\left(q_{\boldsymbol{\bullet}}(\theta), q_{\boldsymbol{*}}(\theta)\right)-w_{\theta+\pi / 2}\left(p_{\bullet}, p_{\bullet}\right) \cdot w_{\theta}\left(p_{\bullet}, p_{\bullet}\right) .
\end{aligned}
$$


Since the four extreme points $q_{*}(\theta), q_{*}(\theta), q_{*}(\theta), q_{*}(\theta)$ are fixed in a primary interval $I \subset[0, \pi / 2)$, the function $a_{E}(\theta)$ over $\theta \in I \cap J$ is indeed a piecewise sinusoidal function of frequency 2 with $O(1)$ breakpoints by Observations 1 and 2 , so can be minimized in $O(1)$ time.

From the observations above, we are ready to describe our algorithm for the minimumarea rectangular annulus problem. First, specify all primary intervals in $O(n \log n)$ time by Toussaint 23 and all MER classes in $O\left(n^{3}\right)$ time using the algorithm by Chaudhuri et al. [11]. For each MER class $E$ with valid interval $J$, we call a primary interval $I$ relevant to $E$ if $I \cap J \neq \emptyset$. We then compute all relevant primary intervals for each MER class in $O\left(n^{3}\right)$ time as follows.

Lemma 10 The number of pairs $(E, I)$ such that $E$ is a MER class and $I$ is a primary interval relevant to $E$ is $O\left(n^{3}\right)$, and all such pairs can be computed in $O\left(n^{3}\right)$ time.

Proof. Let $T$ be the number of such pairs $(E, I)$. Since the algorithm by Chaudhuri et al. [11 runs in an orientation-sweeping fashion, we can easily specify and store all primary intervals $I$ relevant to each MER class $E$ in time $O\left(n^{3}+T\right)$.

Now, we show that $T=O\left(n^{3}\right)$, so the running time above is bounded also by $O\left(n^{3}\right)$. Consider any two consecutive primary intervals $I$ and $I^{\prime}$, and let $\theta$ be the shared endpoint of $I$ and $I^{\prime}$. Naamad et al. 19 showed that the number of maximal empty rectangles in a fixed orientation $\theta$ is $O\left(n^{2}\right)$. This implies that there are at most $O\left(n^{2}\right)$ MER classes $E$ such that both $I$ and $I^{\prime}$ are relevant to $E$. Since there are $O(n)$ primary intervals by Lemma 9 and $O\left(n^{3}\right)$ MER classes shown by Chaudhuri et al. [11, we conclude that $T=O\left(n^{2} \cdot n+n^{3}\right)=O\left(n^{3}\right)$.

Then, for each MER class $E=\left(p_{*}, p_{\bullet}, p_{\bullet}, p_{*} ; J\right)$ and each primary interval $I$ relevant to $E$, we construct the function $a_{E}(\theta)$ and minimize it over $\theta \in I \cap J$ in $O(1)$ time. The minimum of such minima over all MER classes and relevant primary intervals is indeed the minimum possible area of a rectangular annulus enclosing $P$ since $a(\theta)=\min _{E} a_{E}(\theta)$ is the lower envelope of $a_{E}$ over all MER classes $E$ and we want to compute

$$
\min _{\theta \in[0, \pi / 2)} a(\theta)=\min _{\theta \in[0, \pi / 2)} \min _{E} a_{E}(\theta)=\min _{E} \min _{\theta \in[0, \pi / 2)} a_{E}(\theta) .
$$

This proves the correctness of our algorithm. Hence, we conclude the following theorem.

Theorem 9 A minimum-area rectangular annulus enclosing $n$ points over all orientations can be computed in $O\left(n^{3}\right)$ time.

Our algorithm described above indeed can find all minimum-area rectangular annuli enclosing $P$ over all orientations. Therefore, a minimum-width minimum-area rectangular annulus can be found by selecting one with minimum width over all minimum-area annuli.

Corollary 4 A minimum-width minimum-area rectangular annulus enclosing $n$ points over all orientations can be computed in $O\left(n^{3}\right)$ time.

\section{Square Annuli in Arbitrary Orientation}

In this section, we consider the square annulus problem in arbitrary orientation.

Let $a(\theta)$ be the area of a minimum-area $\theta$-aligned square annulus enclosing $P$, and $w(\theta)$ be the width of a minimum-width $\theta$-aligned square annulus enclosing $P$. By Lemma 2 , the outer square of a minimum-area $\theta$-aligned square annulus should be a smallest enclosing $\theta$ aligned square for $P$. Let $d(\theta)$ be the side length of a smallest enclosing $\theta$-aligned square for $P$. We have $d(\theta)=\max \left\{w_{\theta+\pi / 2}\left(q_{*}(\theta), q_{*}(\theta)\right), w_{\theta}\left(q_{*}(\theta), q_{*}(\theta)\right)\right\}$ since a smallest enclosing $\theta$-aligned square for $P$ contains the smallest enclosing $\theta$-aligned rectangle $R_{\theta}$ for $P$. We then have

$$
a(\theta)=4 d(\theta) w(\theta)-4(w(\theta))^{2},
$$




\section{by Lemma 3 .}

The author in 7] proved that the width function $w(\theta)$ on $[0, \pi / 2)$ is piecewise base-0 frequency-1 sinusoidal with $O\left(n^{3}\right)$ breakpoints, and showed how to compute its explicit description in $O\left(n^{3} \log n\right)$ time. Using this, we have the following result.

Lemma 11 The function $a:[0, \pi / 2) \rightarrow \mathbb{R}$ is piecewise frequency-2 sinusoidal with $O\left(n^{3}\right)$ breakpoints, and can be explicitly computed in $O\left(n^{3} \log n\right)$ time.

Proof. As introduced above, by the results in [7], $w:[0, \pi / 2) \rightarrow \mathbb{R}$ is piecewise base-0 frequency1 sinusoidal with $O\left(n^{3}\right)$ breakpoints and can be explicitly computed in $O\left(n^{3} \log n\right)$ time. On the other hand, the function $d:[0, \pi / 2) \rightarrow \mathbb{R}$ is piecewise base- 0 frequency- 1 sinusoidal with $O(n)$ breakpoints by Lemma 9 , since $d$ has at most $O(1)$ breakpoints in each primary interval by Observation 1 .

Now, recall that $a(\theta)=4 d(\theta) w(\theta)-4(w(\theta))^{2}$. So, each breakpoint of $a$ is either a breakpoint of $w$ or one of $d$. Between two consecutive breakpoints of $a$, both $d$ and $w$ are sinusoidal functions of frequency 1 and base 0 . As $a$ is the sum of products of two such sinusoidal functions, it appears to be a sinusoidal function of frequency 2 by Observations 1 and 2 .

After gathering the breakpoints of $w$ and $d$ and sorting them, we can obtain an explicit description of $a$ in $O(1)$ time per breakpoint. Hence, $O\left(n^{3} \log n\right)$ time is sufficient.

This already implies that, in $O\left(n^{3} \log n\right)$ time, we can indeed minimize the area function $a(\theta)$ over $\theta \in[0, \pi / 2)$ and obtain a minimum-area square annulus enclosing $P$ in arbitrary orientation. In the following, we show how to improve this downto $O\left(n^{3}\right)$ without computing a full description of the area function $a$.

For the purpose, we make use of the maximal empty rectangles in arbitrary orientation, as done above for the minimum-area rectangular annulus problem. Our approach here is based on an easy observation that the inner square of a minimum-area $\theta$-aligned square annulus should be contained in a maximal empty $\theta$-aligned rectangle. We thus check each MER class $E=\left(p_{*}, p_{*}, p_{*}, p_{*} ; J\right)$, searching a minimum-area square annulus whose inner square is contained in the maximal empty rectangle $E_{\theta}=\left(p_{\bullet}, p_{\bullet}, p_{*}, p_{;} ; \theta\right)$ for some $\theta \in J$. More precisely, we define $a_{E}(\theta)$ for $\theta \in J$ to be the minimum possible area of square annuli whose outer square is a smallest enclosing $\theta$-aligned square for $P$ and whose inner square is contained in $E_{\theta}$. By definition, possible locations of inner squares are restricted; if there is no possible center for an inner square, then $a_{E}(\theta)$ is defined to be the area of the outer square, that is, $a_{E}(\theta)=(d(\theta))^{2}$. Details are given below.

Let $C_{\theta}$ be the set of centers of all smallest $\theta$-aligned squares enclosing $P$. Observe that $C_{\theta}$ forms a line segment whose orientation is $\theta$ if $d(\theta)=w_{\theta+\pi / 2}\left(q_{*}(\theta), q_{*}(\theta)\right)$, or $\theta+\pi / 2$ if $d(\theta)=$ $w_{\theta}\left(q_{\boldsymbol{*}}(\theta), q_{*}(\theta)\right)$. Further, its length $\left|C_{\theta}\right|$ is equal to $\left|w_{\theta+\pi / 2}\left(q_{\bullet}(\theta), q_{*}(\theta)\right)-w_{\theta}\left(q_{\boldsymbol{*}}(\theta), q_{*}(\theta)\right)\right|$. See Figure 3(a) for an illustration when $d(\theta)=w_{\theta+\pi / 2}\left(q_{*}(\theta), q_{*}(\theta)\right)$, so $C_{\theta}$ is a $\theta$-aligned segment. Lemma 2 implies that any minimum-area $\theta$-aligned square annulus is centered at some $c \in C_{\theta}$, and so is its inner square.

Consider any MER class $E=\left(p_{\bullet}, p_{\bullet}, p_{\bullet}, p_{*} ; J\right)$. Define $\delta_{E}(\theta, c)$ for $\theta \in[0, \pi / 2)$ and $c \in C_{\theta}$ to be the side length of the largest $\theta$-aligned square centered at $c$ contained in $E_{\theta}$ if $\theta \in J$ and $c \in C_{\theta}$, or zero, otherwise. Specifically, it holds that

$$
\delta_{E}(\theta, c):=\left\{\begin{array}{ll}
\min \left\{w_{\theta+\pi / 2}\left(p_{\bullet}, c\right), w_{\theta+\pi / 2}\left(p_{\bullet}, c\right), w_{\theta}\left(p_{\boldsymbol{\bullet}}, c\right), w_{\theta}\left(p_{\boldsymbol{*}}, c\right)\right\} & \text { if } \theta \in J \text { and } c \in C_{\theta} \cap E_{\theta} \\
0 & \text { otherwise }
\end{array} .\right.
$$

See Figure 3(b) for an illustration. We then define $\delta_{E}(\theta):=\max _{c \in C_{\theta}} \delta_{E}(\theta, c)$.

We then consider a primary interval $I \subset[0, \pi / 2)$ and fixes the four extreme points $q_{\text {: }}=q_{\mathrm{m}}(\theta)$ for $\bullet \in\{\bullet, \bullet, \infty\}$. Note that the functions $\delta_{E}(\theta, c)$ and $\delta_{E}(\theta)$ in $I \cap J$ are dependent only on 


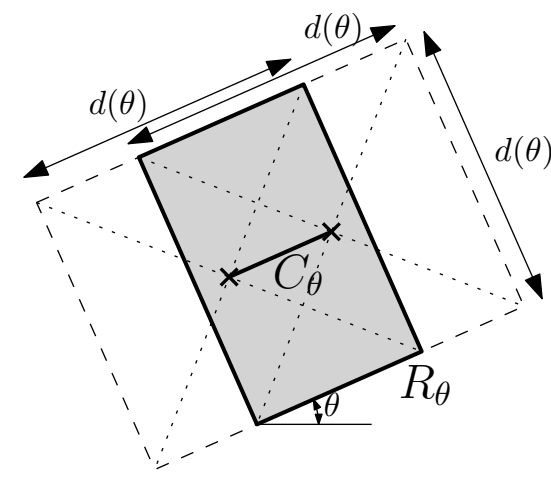

(a)

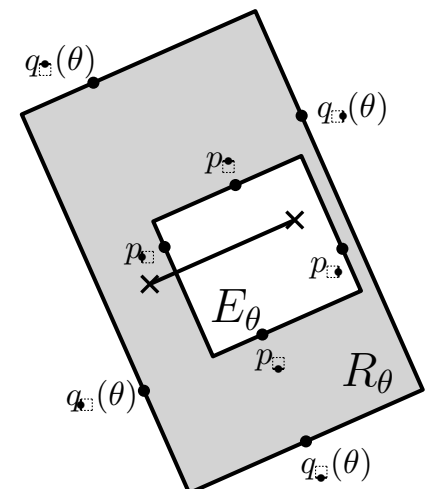

(b)

Figure 3: (a) How $C_{\theta}$ is formed, when it is $\theta$-aligned. Two endpoints of $C_{\theta}$ are the centers of the leftmost and rightmost smallest $\theta$-aligned square enclosing $P$ and thus enclosing $R_{\theta}$. (b) For a MER class $E, E_{\theta}$ is a maximal empty $\theta$-aligned rectangle contained in $R_{\theta}$.

the eight points $q_{*}, q_{\bullet}, q_{\boldsymbol{*}}, q_{*}, p_{\bullet}, p_{\bullet}, p_{\bullet}, p_{\bullet} \in P$, so are of constant complexity. More precisely, we observe the following, while its proof is postponed to Appendix A.

Lemma 12 For any MER class $E$ with valid interval $J$ and any primary interval $I$, the function $\delta_{E}(\theta)$ over $\theta \in I \cap J$ is piecewise base-0 frequency-1 sinusoidal with $O(1)$ breakpoints.

Now, let $a_{E}(\theta):=(d(\theta))^{2}-\left(\delta_{E}(\theta)\right)^{2}$ be the minimum possible area of a square annulus enclosing $P$ whose outer square is a smallest $\theta$-aligned square enclosing $P$ and inner square is contained in $E_{\theta}$. Observe that $a(\theta)=\min _{E} a_{E}(\theta)$ since the inner square of a minimumarea $\theta$-aligned square annulus should be contained in a maximal empty $\theta$-aligned rectangle. In the minimum-area square annulus problem in arbitrary orientation, we want to compute $\min _{\theta \in[0, \pi / 2)} a(\theta)$ and this is equivalent to compute

$$
\min _{\theta \in[0, \pi / 2)} \min _{E} a_{E}(\theta)=\min _{E} \min _{\theta \in[0, \pi / 2)} a_{E}(\theta) .
$$

This indeed describes our algorithm.

Our algorithm runs as follows: First, as done for the rectangular case, we compute all primary intervals and MER classes in $O\left(n^{3}\right)$ time using the algorithm by Chaudhuri et al. [11]. We also specify and store all primary intervals $I$ relevant to each MER class in time $O\left(n^{3}\right)$ by Lemma 10, as done in the previous section. For each MER class $E=\left(p_{*}, p_{\bullet}, p_{\boldsymbol{*}}, p_{;} ; J\right)$ and each primary interval $I$ relevant to $E$, we minimize $a_{E}(\theta)=(d(\theta))^{2}-\left(\delta_{E}(\theta)\right)^{2}$ over $\theta \in I \cap J$. As discussed above and by Lemma 12 , both functions $d(\theta)$ and $\delta_{E}(\theta)$ are piecewise base-0 frequency1 sinusoidal with $O(1)$ breakpoints in $I \cap J$. Thus, the function $a_{E}$ is piecewise frequency-2 sinusoidal with $O(1)$ breakpoints by Observations 1 and 2, so can be minimized in $O(1)$ time. By taking the minimum over those $O\left(n^{3}\right)$ minima we have computed in the above process, we obtain the minimum possible area of any square annulus enclosing $P$ and the corresponding annulus. Therefore, we conclude the following theorem.

Theorem 10 A minimum-area square annulus enclosing $n$ points over all orientations can be computed in $O\left(n^{3}\right)$ time.

The same approach also applies to the minimum-width problem. We define $w_{E}(\theta):=$ $d(\theta) / 2-\delta_{E}(\theta) / 2$, which is the minimum possible width of a square annulus enclosing $P$ whose outer square is a smallest $\theta$-aligned square enclosing $P$ and inner square is contained in $E_{\theta}$. We 
then have $w(\theta)=\min _{E} w_{E}(\theta)$ and want to compute

$$
\min _{\theta \in[0, \pi / 2)} w(\theta)=\min _{\theta \in[0, \pi / 2)} \min _{E} w_{E}(\theta)=\min _{E} \min _{\theta \in[0, \pi / 2)} w_{E}(\theta) .
$$

Thus, to compute the minimum possible width and a corresponding square annulus, we minimize $w_{E}(\theta)=d(\theta) / 2-\delta_{E}(\theta) / 2$ over $\theta \in I \cap J$, for each MER class $E=\left(p_{\star}, p_{\bullet}, p_{*}, p_{*} ; J\right)$ and each primary interval $I$ relevant to $E$. Again by Lemma 12, we deduce that $w_{E}(\theta)$ in $I \cap J$ is a piecewise sinusoidal function of frequency 1 and base 0 with $O(1)$ breakpoints, so can be minimized in $O(1)$ time.

Hence, we also conclude the following.

Theorem 11 A minimum-width square annulus enclosing $n$ points over all orientations can be computed in $O\left(n^{3}\right)$ time.

Note that this even improves the previously fastest algorithm of running time $O\left(n^{3} \log n\right)$ by the author 7 .

Both algorithms for the minimum-width and minimum-area problems can indeed find all minimum-width or minimum-area square annuli enclosing $P$ over all orientations in the same time bound. Therefore, we conclude the following corollary.

Corollary 5 A minimum-width minimum-area or minimum-area minimum-width square annulus enclosing $n$ points over all orientations can be computed in $O\left(n^{3}\right)$ time.

\section{Concluding Remarks}

We have presented first algorithms on the minimum-area square and rectangular annulus problem. On one hand, the minimum-area problem in a fixed orientation is shown to have a close relation with the minimum-width problem and the largest empty rectangle problem, so can be solved by existing algorithms. On the other hand, our algorithms for the problem in arbitrary orientation make use of the concept of maximal empty rectangles among input points. This approach also applies to the problem of computing the largest empty square among $P$ over all orientations. An empty square among $P$ is an empty rectangle among $P$ that is a square.

Theorem 12 Given a set $P$ of $n$ points, a largest empty square among $P$ over all orientations can be computed in $O\left(n^{3}\right)$ time.

Proof. Consider any MER class $E=\left(p_{*}, p_{*}, p_{*}, p_{*} ; J\right)$. Let $s_{E}(\theta)$ be the side length of a largest empty $\theta$-aligned square contained in $E_{\theta}$. Also, let $s(\theta)$ be the side length of a largest empty $\theta$-aligned square among $P$. Then, we have $s(\theta)=\max _{E} s_{E}(\theta)$. Since we want to maximize $s(\theta)$ over $\theta \in[0, \pi / 2)$ and we have

$$
\max _{\theta \in[0, \pi / 2)} s(\theta)=\max _{\theta \in[0, \pi / 2)} \max _{E} s_{E}(\theta)=\max _{E} \max _{\theta \in[0, \pi / 2)} s_{E}(\theta),
$$

this theorem is obtained by maximizing $s_{E}(\theta)$ for each MER class $E$ among the $n$ input points, using the same algorithmic framework as in Theorems 9 and 10.

The last task to be done is to check $s_{E}(\theta)$ is piecewise sinusoidal of frequency 1 and base 0 with $O(1)$ breakpoints. This directly follows from the observation that

$$
s_{E}(\theta)=\min \left\{w_{\theta+\pi / 2}\left(p_{\bullet}, p_{\bullet}\right), w_{\theta}\left(p_{\bullet}, p_{*}\right)\right\}
$$

since it depends only on the four fixed points $p_{\bullet}, p_{\bullet}, p_{\bullet}$, and $p_{*}$.

It is quite surprising that this is the first nontrivial bound for the problem in the literature, to our best knowledge. The problem of computing a largest empty square in a fixed orientation 
can be found in $O(n \log n)$ time by searching the $L_{\infty}$ Voronoi diagram of the input points, as also pointed out in early papers $[5,12,19$.

Our algorithmic results seem to have room for improvement for several cases, in particular, for arbitrary orientation cases. Note that the minimum-area square and (general) rectangular annulus problem in a fixed orientation has a lower bound of $\Omega(n \log n)$ by our reductions shown in Lemmas 3 and 7. In particular, improving the algorithms for the minimum-area rectangular problem automatically improves those for the largest empty rectangle problem. For the problems in arbitrary orientation, there is no known nontrivial lower bound, other than $\Omega(n \log n)$ or $\Omega(n)$ which follows directly from that for the fixed-orientation problem.

Our algorithms for square and rectangular annuli in arbitrary orientation are heavily dependent on the maximal empty rectangles $n$ points. It would be interesting to ask a different approach that avoids computing all maximal empty rectangles, which already takes $O\left(n^{3}\right)$ time. In particular, to compute a minimum-area square annulus or a largest empty square, it is sufficient to check all maximal empty squares among $P$, instead of maximal empty rectangles. Similarly to maximal empty rectangles, a maximal empty square can be defined to be an empty square such that its three sides contain a point in $P$. For a fixed orientation $\theta \in[0, \pi / 2)$, the number of maximal empty squares is bounded by $O(n)$ since each of them defines a vertex of the $L_{\infty}$ Voronoi diagram. However, we are unaware of any result on the number of maximal empty squares over all orientations. Its upper bound is $O\left(n^{3}\right)$ from the number of maximal empty rectangles, and it can be $\Omega\left(n^{2}\right)$ sometimes by a simple construction of $P$. If the correct bound is subcubic, then there would be hope to improve the $O\left(n^{3}\right)$ algorithms for the minimum-area square annulus and the largest empty square problems to subcubic-time algorithms.

\section{References}

[1] M. Abellanas, F. Hurtado, C. Icking, L. Ma, B. Palop, and P. Ramos. Best fitting rectangles. In Proc. Euro. Workshop Comput. Geom. (EuroCG 2003), 2003.

[2] P. Agarwal and M. Sharir. Efficient randomized algorithms for some geometric optimization problems. Discrete Comput. Geom., 16:317-337, 1996.

[3] P. Agarwal, M. Sharir, and S. Toledo. Applications of parametric searching in geometric optimization. J. Algo., 17:292-318, 1994.

[4] P. K. Agarwal, S. Har-Peled, and K. R. Varadarajan. Approximating extent measures of points. J. ACM, 51(4):606-635, 2004.

[5] A. Aggarwal and S. Suri. Fast algorithm for computing the largest empty rectangle. In Proc. 3rd ACM Sympos. Comput. Geom. (SoCG 1987), pages 278-290, 1987.

[6] H.-K. Ahn, T. Ahn, S. W. Bae, J. Choi, M. Kim, E. Oh, C.-S. Shin, and S. D. Yoon. Minimum-width annulus with outliers: Circular, square, and rectangular cases. Infor. Proc. Lett., 145:16-23, 2019.

[7] S. W. Bae. Computing a minimum-width square annulus in arbitrary orientation. Theoret. Comput. Sci., 718:2-13, 2018.

[8] S. W. Bae. Computing a minimum-width square or rectangular annulus with outliers. Comput. Geom.: Theory Appl., 76:33-45, 2019.

[9] S. W. Bae, A. Baral, and P. R. Sinha Mahapatra. Maximum-width empty square and rectangular annulus. In Proc. 13th Int. Conf. Workshop Alg. Comput. (WALCOM 2019), volume 11355 of $L N C S$, pages 69-81, 2019. 
[10] T. Chan. Approximating the diameter, width, smallest enclosing cylinder, and minimumwidth annulus. Int. J. Comput. Geom. Appl., 12:67-85, 2002.

[11] J. Chaudhuri, S. C. Nandy, and S. Das. Largest empty rectangle among a point set. J. Algo., 46:54-78, 2003.

[12] B. Chazelle, R. Drysdale, and D. Lee. Computing the largest empty rectangle. SIAM J. Comput., 15:300-315, 1986.

[13] J. M. Díz-Báñez, F. Hurtado, H. Meijer, D. Rappaport, and J. A. Sellarès. The largest empty annulus problem. Int. J. Comput. Geom. Appl., 13(4):317-325, 2003.

[14] H. Ebara, N. Fukuyama, H. Nakano, and Y. Nakanishi. Roundness algorithms using the Voronoi diagrams. In Abstracts 1st Canadian Conf. Comput. Geom (CCCG), page 41, 1989.

[15] O. N. Gluchshenko, H. W. Hamacher, and A. Tamir. An optimal $O(n \log n)$ algorithm for finding an enclosing planar rectilinear annulus of minimum width. Operations Research Lett., 37(3):168-170, 2009.

[16] J. Hershberger. Finding the upper envelope of $n$ line segments in $O(n \log n)$ time. Inform. Proc. Lett., 33:169-174, 1989.

[17] M. Mckenna, J. O'Rourke, and S. Suri. Finding the largest rectangle in an orthogonal polygon. In Proc. 23rd Annual Allerton Conf. Comm. Control Comput., 1985.

[18] J. Mukherjee, P. Mahapatra, A. Karmakar, and S. Das. Minimum-width rectangular annulus. Theoretical Comput. Sci., 508:74-80, 2013.

[19] A. Naamad, D. Lee, and W. Hsu. On the maximum empty rectangle problem. Discrete Appl. Math., 8:267-277, 1984.

[20] M. Orlowski. A new algorithm for largest empty rectangle problem. Algorithmica, 5:65-73, 1990.

[21] U. Roy and X. Zhang. Establishment of a pair of concentric circles with the minimum radial separation for assessing roundness error. Computer-Aided Design, 24(3):161-168, 1992 .

[22] M. Sharir and P. K. Agarwal. Davenport-Schinzel Sequences and Their Geometric Applications. Cambridge University Press, New York, 1995.

[23] G. Toussaint. Solving geometric problems with the rotating calipers. In Proc. IEEE MELECON, 1983.

[24] A. Wainstein. A non-monotonous placement problem in the plane. In Software Systems for Solving Optimal Planning Problems, Abstract: 9th All-Union Symp. USSR, Symp., pages 70-71, 1986.

\section{A Proof of Lemma 12}

In this Appendix, we give a proof of Lemma 12 .

Lemma 12 For any MER class $E$ with valid interval $J$ and any primary interval $I$, the function $\delta_{E}(\theta)$ over $\theta \in I \cap J$ is piecewise base-0 frequency-1 sinusoidal with $O(1)$ breakpoints. 


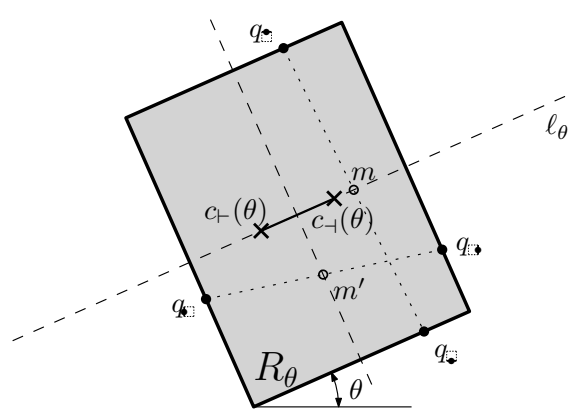

(a)

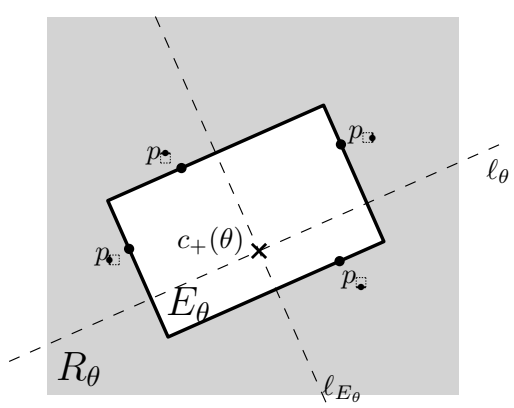

(b)

Figure 4: How (a) $c_{\vdash}(\theta)$ and $c_{\dashv}(\theta)$, and (b) $c_{+}(\theta)$ are determined. $\ell_{E_{\theta}}$ is the $(\theta+\pi / 2)$-aligned line halving $E_{\theta}$.

We first recall some definitions: $R_{\theta}$ is the smallest $\theta$-aligned enclosing rectangle for $P$; $C_{\theta}$ is the set of centers of all smallest $\theta$-aligned enclosing squares for $P ; d(\theta)=\max \left\{w_{\theta+\pi / 2}\left(q_{*}(\theta), q_{*}(\theta)\right)\right.$, $\left.w_{\theta}\left(q_{\text {: }}(\theta), q_{\text {: }}(\theta)\right)\right\}$ is the side length of a smallest $\theta$-aligned enclosing square for $P$; For each MER class $E=\left(p_{*}, p_{*}, p_{*}, p_{*} ; J\right)$, and for any $\theta \in[0, \pi / 2)$ and any $c \in C_{\theta}$

$$
\delta_{E}(\theta, c)= \begin{cases}\min \left\{w_{\theta+\pi / 2}\left(p_{\bullet}, c\right), w_{\theta+\pi / 2}\left(p_{*}, c\right), w_{\theta}\left(p_{\bullet}, c\right), w_{\theta}\left(p_{*}, c\right)\right\} & \theta \in J, c \in C_{\theta} \cap E_{\theta} \\ 0 & \text { otherwise }\end{cases}
$$

is the side length of the largest $\theta$-aligned square centered at $c$ contained in $E_{\theta}$ if $\theta \in J$ and $c \in C_{\theta}$, or zero, otherwise; lastly, $\delta_{E}(\theta)=\max _{c \in C_{\theta}} \delta_{E}(\theta, c)$.

Here, for convenience, we refine primary intervals as follows. Throughout this proof, a primary interval is redefined to be a maximal interval $I \subset[0, \pi / 2)$ such that the four extreme points are fixed, so $q_{*}=q_{*}(\theta), q_{*}=q_{*}(\theta), q_{* *}=q_{*:}(\theta)$, and $q_{*}=q_{*}(\theta)$ over all $\theta \in I$, and further there is no $\theta \in I$ with $w_{\theta+\pi / 2}\left(q_{*}, q_{*}\right)=w_{\theta}\left(q_{*}, q_{*}\right)$. The second condition keeps the function $d(\theta)$ to be in a simpler form in a primary interval: $d(\theta)=w_{\theta+\pi / 2}\left(q_{\bullet}, q_{\bullet}\right)$ or $d(\theta)=w_{\theta}\left(q_{\bullet}, q_{*}\right)$. For a fixed tuple of $\left(q_{*}, q_{*}, q_{*}, q_{*}\right)$, there is at most one $\theta$ such that $w_{\theta+\pi / 2}\left(q_{*}, q_{*}\right)=w_{\theta}\left(q_{*}, q_{*}\right)$ by Observation 1. Hence, in order to prove the lemma, it suffices to consider each of these refined primary interval.

Now, we give a proof for the lemma. Consider any MER class $E=\left(p_{\bullet}, p_{\bullet}, p_{\bullet}, p_{*} ; J\right)$ and a (refined) primary interval $I$. If $I \cap J=\emptyset$, then the lemma follows trivially, so suppose in the following that $I \cap J \neq \emptyset$. Let $q_{*}=q_{*}(\theta), q_{*}=q_{*}(\theta), q_{*}=q_{*}(\theta)$, and $q_{*}=q_{*}(\theta)$ for any $\theta \in I$. Without loss of generality, we assume that $w_{\theta+\pi / 2}\left(q_{\bullet}, q_{\bullet}\right) \geq w_{\theta}\left(q_{\bullet}, q_{*}\right)$ for any $\theta \in I$, so $d(\theta)=w_{\theta+\pi / 2}\left(q_{\bullet}, q_{*}\right)$. Then, $C_{\theta}$ forms a $\theta$-aligned line segment whose midpoint is located at the center of $R_{\theta}$, that is, the intersection of two diagonals of $R_{\theta}$, and whose length is $\left|C_{\theta}\right|=w_{\theta+\pi / 2}\left(q_{*}, q_{*}\right)-w_{\theta}\left(q_{*}, q_{*}\right)$.

Let $\ell_{\theta}$ be the line extending $C_{\theta}$. We let $m$ be the midpoint of line segment $\overline{q_{\bullet} q_{*}}$ and $m^{\prime}$ be the midpoint of line segment $\overline{q_{k} q_{*}}$. Note that the $\theta$-aligned line through $m$ horizontally halves $R_{\theta}$ and the $(\theta+\pi / 2)$-aligned line through $m^{\prime}$ vertically halves $R_{\theta}$. Observe that $\ell_{\theta}$ is the $\theta$-aligned line through $m$ for any $\theta \in I$, so $\ell_{\theta}$ rotates around $m$ as $\theta \in I$ continuously increases. Also, we denote by $c_{\vdash}(\theta)$ and $c_{\dashv}(\theta)$ the left and right endpoints of $C_{\theta}$ for $\theta \in I$, and let $c_{+}(\theta) \in \ell_{\theta}$ be the intersection point between $\ell_{\theta}$ and the $(\theta+\pi / 2)$-aligned line that halves $R_{\theta}$. See Figure 4 .

We are interested in three empty squares centered at $c_{\vdash}(\theta), c_{\dashv}(\theta)$, and $c_{+}(\theta)$, respectively, that are contained in $E_{\theta}$. Define

$$
\rho_{\vdash}(\theta):=\delta_{E}\left(\theta, c_{\vdash}(\theta)\right), \quad \rho_{\dashv}(\theta):=\delta_{E}\left(\theta, c_{\dashv}(\theta)\right), \quad \text { and } \quad \rho_{+}(\theta):=\delta_{E}\left(\theta, c_{+}(\theta)\right)
$$




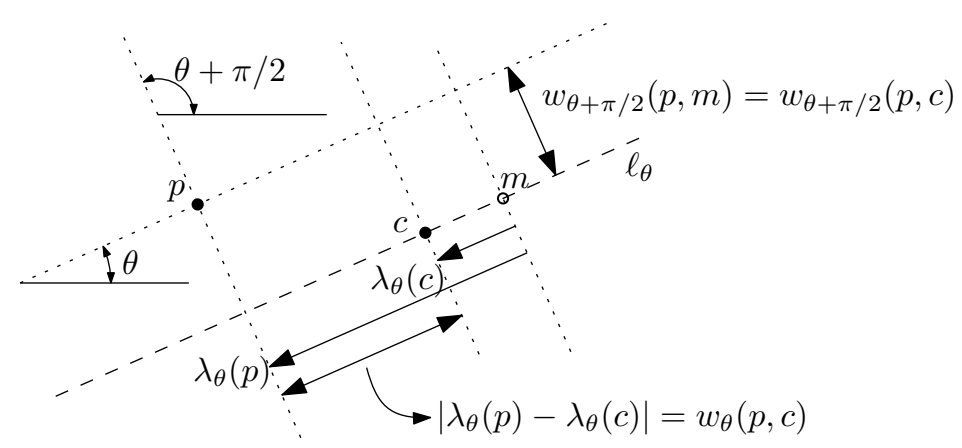

Figure 5: Illustration to the proof of Observation 3 .

to be the side lengths of the largest $\theta$-aligned squares contained in $E_{\theta}$ centered at $c_{\vdash}(\theta), c_{\dashv}(\theta)$, and $c_{+}(\theta)$, respectively. We claim that these three functions over $\theta \in I \cap J$ are all piecewise frequency-1 base-0 sinusoidal. For the purpose, we need more observations. For any $p \in \mathbb{R}^{2}$, let $\lambda_{\theta}(p)$ be the signed distance from $m$ to the orthogonal projection of $p$ onto $\ell_{\theta}$ so that it is positive if $p$ is to the right of $m$ or negative if $p$ is to the left of $m$. Specifically, we have

$$
\lambda_{\theta}(p)=|\overline{p m}| \cdot \cos \left(\theta_{p m}-\theta\right),
$$

where $\theta_{p m} \in[0, \pi)$ is the orientation of segment $\overline{p m}$. Note that $w_{\theta}(p, m)=\left|\lambda_{\theta}(p)\right|$.

We then observe the following.

Observation 3 For any $\theta \in I \cap J$, any $c \in \ell_{\theta}$ and any $p \in \mathbb{R}^{2}$, it holds that

- $w_{\theta+\pi / 2}(p, c)=w_{\theta+\pi / 2}(p, m)$, and

- $w_{\theta}(p, c)=\left|\lambda_{\theta}(p)-\lambda_{\theta}(c)\right|$.

Proof. See Figure 5 for an illustration. Since $c \in \ell_{\theta}$, the distance between the orthogonal projections of $c$ and any $p \in \mathbb{R}^{2}$ onto a $(\theta+\pi / 2)$-aligned line is equal to that between the projections of $m$ and $p$. This proves the first equation.

The value of $w_{\theta}(p, c)$ is equal to the distance between the orthogonal projections of $p$ and $c$ onto $\ell_{\theta}$, and hence is represented by the difference of their relative distances to $m$, which is $\left|\lambda_{\theta}(p)-\lambda_{\theta}(c)\right|$.

Applying the above observation to the case where $c=c_{\vdash}(\theta)$ or $c=c_{\dashv}(\theta)$, we obtain the following.

Observation 4 For any $\theta \in I \cap J$ and any $p \in \mathbb{R}^{2}$, it holds that

- $w_{\theta+\pi / 2}\left(p, c_{\vdash}(\theta)\right)=w_{\theta+\pi / 2}\left(p, c_{\dashv}(\theta)\right)=w_{\theta+\pi / 2}(p, m)$,

- $w_{\theta}\left(p, c_{\vdash}(\theta)\right)=\left|\lambda_{\theta}(p)-\left(\lambda_{\theta}\left(m^{\prime}\right)-\frac{1}{2}\left(w_{\theta+\pi / 2}\left(q_{*}, q_{*}\right)-w_{\theta}\left(q_{*}, q_{*}\right)\right)\right)\right|$, and

- $w_{\theta}\left(p, c_{\dashv}(\theta)\right)=\left|\lambda_{\theta}(p)-\left(\lambda_{\theta}\left(m^{\prime}\right)+\frac{1}{2}\left(w_{\theta+\pi / 2}\left(q_{\bullet}, q_{*}\right)-w_{\theta}\left(q_{\bullet}, q_{*}\right)\right)\right)\right|$.

Proof. The first equation directly follows from Observation 3 when $c=c_{\vdash}(\theta)$ and $c=c_{\dashv}(\theta)$.

To see the last two, recall that $C_{\theta} \subset \ell_{\theta}$ is a line segment of length $w_{\theta+\pi / 2}\left(q_{\bullet}, q_{\bullet}\right)-w_{\theta}\left(q_{\bullet}, q_{*}\right)$, and its midpoint is the intersection of $\ell_{\theta}$ and the $(\theta+\pi / 2)$-aligned line through $m^{\prime}$. Thus, we have

$$
\lambda_{\theta}\left(c_{\vdash}(\theta)\right)=\lambda_{\theta}\left(m^{\prime}\right)-\left|C_{\theta}\right| / 2, \quad \text { and } \quad \lambda_{\theta}\left(c_{\dashv}(\theta)\right)=\lambda_{\theta}\left(m^{\prime}\right)+\left|C_{\theta}\right| / 2,
$$

where $\left|C_{\theta}\right|=w_{\theta+\pi / 2}\left(q_{*}, q_{*}\right)-w_{\theta}\left(q_{*}, q_{*}\right)$ is the length of segment $C_{\theta}$. Plugging these into Observation 3, we obtain the last two equations.

Similarly, for the case $c=c_{+}(\theta)$, we observe the following. 
Observation 5 For any $\theta \in I \cap J$ and any $p \in \mathbb{R}^{2}$, it holds that

- $w_{\theta+\pi / 2}\left(p, c_{+}(\theta)\right)=w_{\theta+\pi / 2}(p, m)$, and

- $w_{\theta}\left(p, c_{+}(\theta)\right)=\left|\lambda_{\theta}(p)-\frac{1}{2}\left(\lambda_{\theta}\left(p_{*}\right)+\lambda_{\theta}\left(p_{\boldsymbol{*}}\right)\right)\right|$.

Proof. This follows from Observation 3 and the fact that $\lambda_{\theta}\left(c_{+}(\theta)\right)=\frac{1}{2}\left(\lambda_{\theta}\left(p_{\$}\right)+\lambda_{\theta}\left(p_{\bullet}\right)\right)$.

From Observations 4 and 5 , it is easy to see that the three functions $\rho_{\vdash}, \rho_{\dashv}$, and $\rho_{+}$behave nicely.

Lemma 13 The three functions $\rho_{\vdash}(\theta), \rho_{\dashv}(\theta)$, and $\rho_{+}(\theta)$ over $\theta \in I \cap J$ are piecewise frequency-1 base-0 sinusoidal with $O(1)$ breakpoints.

Proof. First, consider the function $\rho_{\vdash}(\theta)=\delta_{E}\left(\theta, c_{\vdash}(\theta)\right)$. By definition, we have

$$
\rho_{\vdash}(\theta)=\min \left\{w_{\theta+\pi / 2}\left(p_{\bullet}, c_{\vdash}(\theta)\right), w_{\theta+\pi / 2}\left(p_{\bullet}, c_{\vdash}(\theta)\right), w_{\theta}\left(p_{\bullet}, c_{\vdash}(\theta)\right), w_{\theta}\left(p_{\bullet}, c_{\vdash}(\theta)\right)\right\},
$$

if $c_{\vdash}(\theta) \in E_{\theta}$, and $\rho_{\vdash}(\theta)=0$, otherwise. In case of $c_{\vdash}(\theta) \in E_{\theta}$, Observation 4 implies that

$$
\begin{aligned}
\rho_{\vdash}(\theta)=\min \left\{w_{\theta+\pi / 2}\left(p_{\bullet}, m\right),\right. & w_{\theta+\pi / 2}\left(p_{\bullet}, m\right), \\
& \left.\left|\lambda_{\theta}\left(p_{\bullet}\right)-\left(\lambda_{\theta}\left(m^{\prime}\right)-\left|C_{\theta}\right| / 2\right)\right|,\left|\lambda_{\theta}\left(p_{\bullet}\right)-\left(\lambda_{\theta}\left(m^{\prime}\right)-\left|C_{\theta}\right| / 2\right)\right|\right\},
\end{aligned}
$$

where $\left|C_{\theta}\right|=w_{\theta+\pi / 2}\left(q_{*}, q_{*}\right)-w_{\theta}\left(q_{\boldsymbol{*}}, q_{\boldsymbol{*}}\right)$ is the length of $C_{\theta}$. Now, we check the four terms in the $\min \{\cdot\}$. The first two are obviously piecewise frequency- 1 base- 0 sinusoidal. For the last two, observe that $\lambda_{\theta}(p)$ for any fixed point $p \in \mathbb{R}^{2}$ is frequency- 1 base- 0 sinusoidal by definition, and $\left|C_{\theta}\right|$ is also piecewise frequency- 1 base- 0 sinusoidal. Thus, the last two terms are also piecewise frequency- 1 base- 0 sinusoidal by Observation 1. Hence, $\rho_{\vdash}(\theta)$ is piecewise frequency-1 base- 0 sinusoidal. The number of breakpoints of $\rho_{\vdash}$ is bounded by $O(1)$ since it depends only on a set of constantly many fixed points, namely, $\left\{q_{\bullet}, q_{*}, q_{\boldsymbol{*}}, q_{*}, p_{\star}, p_{*}, p_{\bullet}, p_{*}, m, m^{\prime}\right\}$. The proof for $\rho_{\dashv}(\theta)$ is almost identical to above.

Now, consider the function $\rho_{+}(\theta)=\delta_{E}\left(\theta, c_{+}(\theta)\right)$. By definition, we have

$$
\rho_{+}(\theta)=\min \left\{w_{\theta+\pi / 2}\left(p_{\bullet}, c_{+}(\theta)\right), w_{\theta+\pi / 2}\left(p_{\bullet}, c_{+}(\theta)\right), w_{\theta}\left(p_{\bullet}, c_{+}(\theta)\right), w_{\theta}\left(p_{:}, c_{+}(\theta)\right)\right\},
$$

if $c_{+}(\theta) \in E_{\theta} \cap C_{\theta}$, and $\rho_{+}(\theta)=0$, otherwise. For the case where $c_{+}(\theta) \in E_{\theta} \cap C_{\theta}$, Observation 5 implies that

$$
\rho_{+}(\theta)=\min \left\{w_{\theta+\pi / 2}\left(p_{\bullet}, m\right), w_{\theta+\pi / 2}\left(p_{\bullet}, m\right), \frac{1}{2}\left(\lambda_{\theta}\left(p_{\bullet}\right)-\lambda_{\theta}\left(p_{\bullet}\right)\right)\right\}
$$

By a similar argument as above, $\rho_{+}(\theta)$ is also piecewise frequency-1 base- 0 sinusoidal with $O(1)$ breakpoints.

We then show that the maximum $\delta_{E}(\theta)$ of $\delta_{E}(\theta, c)$ over $c \in E_{\theta} \cap C_{\theta}$, for each $\theta \in I \cap J$, is represented by the three functions $\rho_{\vdash}, \rho_{\dashv}$, and $\rho_{+}$.

Lemma 14 The function $\delta_{E}(\theta)$ over $\theta \in I \cap J$ is represented as follows:

$$
\delta_{E}(\theta)= \begin{cases}0 & C_{\theta} \cap E_{\theta}=\emptyset \\ \rho_{+}(\theta) & c_{+}(\theta) \in E_{\theta} \cap C_{\theta} \\ \rho_{\vdash}(\theta) & c_{+}(\theta) \notin E_{\theta} \cap C_{\theta}, c_{\vdash}(\theta) \in E_{\theta}, c_{\dashv}(\theta) \notin E_{\theta} . \\ \rho_{\dashv}(\theta) & c_{+}(\theta) \notin E_{\theta} \cap C_{\theta}, c_{\vdash}(\theta) \notin E_{\theta}, c_{\dashv}(\theta) \in E_{\theta} \\ \max \left\{\rho_{\vdash}(\theta), \rho_{\dashv}(\theta)\right\} & c_{+}(\theta) \notin E_{\theta} \cap C_{\theta}, C_{\theta} \subset E_{\theta}\end{cases}
$$

Proof. Recall that $\delta_{E}(\theta)=\max _{c \in C_{\theta}} \delta_{E}(\theta, c)$, and $\delta_{E}(\theta, c)=\min \left\{w_{\theta+\pi / 2}\left(p_{\bullet}, c\right), w_{\theta+\pi / 2}\left(p_{\bullet}, c\right)\right.$, $\left.w_{\theta}\left(p_{\boldsymbol{\bullet}}, c\right), w_{\theta}\left(p_{\mathrm{s}}, c\right)\right\}$ if $c \in C_{\theta} \cap E_{\theta}$, or zero, otherwise. For $c \in \ell_{\theta} \cap E_{\theta}$, note that 
- $w_{\theta+\pi / 2}\left(p_{\bullet}, c\right)=w_{\theta+\pi / 2}\left(p_{\bullet}, m\right)$,

- $w_{\theta+\pi / 2}\left(p_{*}, c\right)=w_{\theta+\pi / 2}\left(p_{\bullet}, m\right)$,

- $w_{\theta}\left(p_{\boldsymbol{\theta}}, c\right)=\lambda_{\theta}(c)-\lambda_{\theta}\left(p_{\boldsymbol{*}}\right)$, and

- $w_{\theta}\left(p_{\text {: }}, c\right)=\lambda_{\theta}\left(p_{\bullet \bullet}\right)-\lambda_{\theta}(c)$,

by Observation 3. Hence, for a fixed $\theta \in I \cap J$, the first two terms are constant over all $c \in C_{\theta} \cap E_{\theta}$, and the last two terms are linear functions of $\lambda_{\theta}(c)$ of slope 1 and -1 . This implies that the function $c \mapsto \min \left\{w_{\theta+\pi / 2}\left(p_{\star}, c\right), w_{\theta+\pi / 2}\left(p_{*}, c\right), w_{\theta}\left(p_{\boldsymbol{\bullet}}, c\right), w_{\theta}\left(p_{\star}, c\right)\right\}$ on $c \in \ell_{\theta} \cap E_{\theta}$ is piecewise linear with three pieces whose slopes are 1,0 , and -1 in this order. Observe that the maximum of this function over $c \in \ell_{\theta} \cap E_{\theta}$ is always attained at $c=c_{+}(\theta)$ since

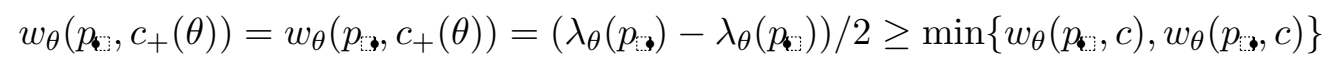

for any $c \in \ell_{\theta} \cap E_{\theta}$.

Now, consider the function $\delta_{E}(\theta, c)$ and its maximum $\delta_{E}(\theta)$ over $c \in C_{\theta} \cap E_{\theta}$. We handle the five cases separately. For the first case where $C_{\theta} \cap E_{\theta}=\emptyset$ it is trivial that $\delta_{E}(\theta)=0$.

Second, assume that $c_{+}(\theta) \in E_{\theta} \cap C_{\theta}$. Then, by the above discussion, we have $\delta_{E}(\theta)=$ $\delta_{E}\left(\theta, c_{+}(\theta)\right)=\rho_{+}(\theta)$.

Next, suppose that $C_{\theta} \cap E_{\theta} \neq \emptyset$ and $c_{+}(\theta) \notin E_{\theta} \cap C_{\theta}$. Then, again by the above discussion, we have either $\delta_{E}(\theta)=\delta_{E}\left(\theta, c_{\vdash}(\theta)\right)$ or $\delta_{E}(\theta)=\delta_{E}\left(\theta, c_{\dashv}(\theta)\right)$. On the other hand, at least one of $c_{\vdash}(\theta)$ or $c_{\dashv}(\theta)$ must be contained in $E_{\theta}$, since $C_{\theta}$ is a $\theta$-aligned line segment and $c_{+}(\theta) \notin C_{\theta}$. Thus,

- if $c_{\dashv}(\theta) \notin E_{\theta}$, then $\delta_{E}(\theta)=\delta_{E}\left(\theta, c_{\vdash}(\theta)\right)=\rho_{\vdash}(\theta)$;

- if $c_{\vdash}(\theta) \notin E(\theta)$, then $\delta_{E}(\theta)=\delta_{E}\left(\theta, c_{\dashv}(\theta)\right)=\rho_{\dashv}(\theta)$;

- otherwise, if both $c_{\vdash}(\theta)$ and $c_{\dashv}(\theta)$ lie in $E_{\theta}$, then we have

$$
\delta_{E}(\theta)=\max \left\{\delta_{E}\left(\theta, c_{\vdash}(\theta)\right), \delta_{E}\left(\theta, c_{\dashv}(\theta)\right\}=\max \left\{\rho_{\vdash}(\theta), \rho_{\dashv}(\theta)\right)\right\} .
$$

This completes the proof of the lemma.

Note that each of $\rho_{\vdash}(\theta), \rho_{\dashv}(\theta)$, and $\rho_{+}(\theta)$ are piecewise sinusoidal of frequency 1 and base 0 as observed in Lemma 13. Hence, by Lemma 14, we verify that $\delta_{E}(\theta)$ is piecewise sinusoidal of frequency 1 and base 0 , too. The number of breakpoints of $\delta_{E}(\theta)$ over $\theta \in I \cap J$ is also bounded by a constant as follows:

The function $\delta_{E}(\theta)$ has the five cases as described in Lemma 14. As $\theta$ continuously increases in $I \cap J$, we turn into a different case from one whenever one of the following events occurs:

(i) When $c_{\vdash}(\theta)$ or $c_{\dashv}(\theta)$ lies on the left or right side of $E_{\theta}$, equivalently, when either one of the following becomes zero: $w_{\theta}\left(p_{\boldsymbol{\bullet}}, c_{\vdash}(\theta)\right), w_{\theta}\left(p_{\boldsymbol{\bullet}}, c_{\dashv}(\theta)\right), w_{\theta}\left(p_{\mathfrak{:} ;}, c_{\vdash}(\theta)\right)$, and $w_{\theta}\left(p_{: \mathbf{s}}, c_{\dashv}(\theta)\right)$.

(ii) When the top or bottom side of $E_{\theta}$ lies on $\ell_{\theta}$, equivalently, when either $w_{\theta+\pi / 2}\left(p_{\bullet}, m\right)=0$ or $w_{\theta+\pi / 2}\left(p_{*}, m\right)=0$.

(iii) When either $c_{\vdash}(\theta)=c_{+}(\theta)$ or $c_{\dashv}(\theta)=c_{+}(\theta)$, equivalently, when either $w_{\theta}\left(p_{\bullet}, c_{\vdash}(\theta)\right)=$ $w_{\theta}\left(p_{:}, c_{\vdash}(\theta)\right)$ or $w_{\theta}\left(p_{\bullet}, c_{\dashv}(\theta)\right)=w_{\theta}\left(p_{\xi}, c_{\dashv}(\theta)\right)$.

As described above, each event occurs when some sinusoidal function of frequency 1 and base 0 becomes zero, so there are a constantly many number of such events by Observation 1 .

This completes the proof of Lemma 12 . 